K. Yamamoto

Nagoya Math. J.

Vol. 116 (1989), 25-42

\title{
ELASTIC WAVES IN TWO SOLIDS AS PROPAGATION OF SINGULARITIES PHENOMENON
}

\author{
KAZUHIRO YAMAMOTO
}

In this paper we shall study elastic waves in two isotropic media with different densities and Lamè's constants. In seismology elastic waves are studied when the border of two media is a hyperplane, however there are no results on elastic waves in the non-flat border case. First in Section 1 we shall show an existence theorem of the solutions of an initial boundary value problem which is satisfied by the displacements of two media. Next we shall discuss about propagation of singularities of the solutions, for Hörmander and Lax-Nirenberg showed that an appearance of propagation of singularities is similar to one of propagation of waves.

As first part on singularities in Section 3 we shall show existence of Stoneley waves as propagation of singularities, which is explained as follows: if the boundary values of the initial data of the solutions have singularities, then there exist singularities of the solutions which start from the singularities of the initial data and propagate in the elliptic region of the border of two media according to the passage of time.

Second part on singularities is to study relations between incident waves and reflected and refracted waves, which are stated in Sections 4, 5 . Under various conditions on the incident angle of singularities corresponding to the fast waves or the slow waves we have interesting refractive phenomena. For example if we assume that densities and Lamè's constants satisfy some conditions, and that the incident angle of singularities corresponding to the fast waves is not sharp, then the solutions have only refracted singularities corresponding to the slow waves (see Theorem 4.1). If the singularities corresponding to the slow waves make incidence, the solutions have both refracted singularities corresponding to the fast and slow waves or they have only refracted singularities cor-

Received September 22, 1987. 
responding to the slow waves (see Theorems 5.1, 5.2).

\section{$\S 1$. Existence of solutions}

In this section we shall show existence of the solutions of initial boundary value problems in two solids. We suppose that there exist two simply connected open subsets $O_{i}(i=1,2)$ of $R^{3}$ with smooth boundaries $\partial O_{i}$ such that $\partial O_{i}$ is compact, or $\partial O_{i}$ is equal to a hyperplane in $\{x:|x|>R\}$ for some positive constant $R$. Then domains $\Omega_{1}, \Omega_{2}$ of two solids are defined as follows; $\Omega_{1}=O_{1} \cap O_{2}$ and $\Omega_{2}=O_{1} \backslash \bar{O}_{2}$. The strain tensors $\varepsilon_{j k}(u)$ $(j, k=1,2,3)$ are $\left(\partial u_{j} / \partial x_{k}+\partial u_{k} / \partial x_{j}\right) / 2$ and in the stress tensors $\sigma_{j k}^{(i)}(u)$ are $\lambda_{i}(\operatorname{div} u) \delta_{j k}+2 \mu_{i} \varepsilon_{j k}(u)$, where $u={ }^{t}\left(u_{1}, u_{2}, u_{3}\right)$ and $\lambda_{i}$ and $\mu_{i}$ are Lamè constants such that $\mu_{i}, 3 \lambda_{i}+2 \mu_{i}$ and $\lambda_{i}+\mu_{i}$ are positive. If the solids are isotropic, then the displacements $u_{i}(x, t)={ }^{t}\left(u_{i 1}, u_{i 2}, u_{i 3}\right)$ in $\Omega_{i}$ satisfy the following boundary value problem:

$$
\begin{aligned}
& \rho_{i} \partial^{2} u_{i j} / \partial t^{2}-\sum_{k=1}^{3} \partial\left(\sigma_{j k}^{(i)}\left(u_{i}\right)\right) ! \partial x_{k}=0 \quad \text { in } \Omega_{i} \times R, \\
& u_{1}=u_{2} \quad \text { on } \Gamma \times R, \\
& \sum_{j} n_{j}(x) \sigma_{j k}^{(1)}\left(u_{1}\right)=\sum_{j} n_{j}(x) \sigma_{j k}^{(2)}\left(u_{2}\right) \quad \text { on } \Gamma \times R, \\
& \sum_{j} n_{j}(x) \sigma_{j k}^{(i)}\left(u_{i}\right)=0 \quad \text { on } \Gamma_{i} \times R,
\end{aligned}
$$

where $\rho_{i}>0$ is the density of $\Omega_{i}, \Gamma=\partial \Omega_{1} \cap \partial \Omega_{2}, \Gamma_{i}=\partial \Omega_{i} \backslash \Gamma$, and $n(x)=$ ${ }^{t}\left(n_{1}(x), n_{2}(x), n_{3}(x)\right)$ is the unit normal vector of $\Gamma$ or $\Gamma_{j}(j=1,2)$.

We consider an initial boundary value problem (1.1) to (1.4) with data

$$
u_{i}(x, 0)=f_{i}(x), \quad\left(\partial u_{i} / \partial t\right)(x, 0)=g_{i}(x) \quad \text { in } \Omega_{i} .
$$

We introduce a Hilbert space $\mathscr{H}$ whose elements are equal to $\left(L^{2}\left(O_{1}\right)\right)^{3}$, with the inner product $(f, g)_{\mathscr{H}}=\rho_{1}(f, g)_{L^{2}\left(\Omega_{2}\right)}+\rho_{2}(f, g)_{L^{2}\left(\Omega_{2}\right)}$, and a subspace $D$ of $\mathscr{H}$ such that $f$ belongs to $D$ if $f \in\left(H^{1}\left(O_{i}\right)\right)^{3}$ and the distributions $\left.L_{i}(f)=\left.\left(\sum_{k} \partial\left(\sigma_{j k}^{(i)}(f)\right) / \partial x_{k}\right)\right|_{a_{i}}\right)_{j=1,2,3}$ belong to $\left(L^{2}\left(\Omega_{i}\right)\right)^{3}$. The boundary conditions (1.3) and (1.4) are represented as follows:

$$
\begin{aligned}
& \int_{\Omega_{1}}\left\{L_{1}(f) \cdot v+\sum_{j, k} \sigma_{k j}^{(1)}(f) \partial v_{k} / \partial x_{j}\right\} d x \\
& \quad+\int_{\Omega_{2}}\left\{L_{2}(f) \cdot v+\sum_{j, k} \sigma_{k j}^{(2)}(f) \partial v_{k} / \partial x_{j}\right\} d x=0
\end{aligned}
$$

for any $v={ }^{t}\left(v_{1}, v_{2}, v_{3}\right) \in\left(H^{1}\left(O_{1}\right)\right)^{3}$. The operator $A$ on $D_{N}=\{f \in D$ : $f$ satisfies (1.6) $\}$ is defined by $\left.A f\right|_{\Omega_{i}}=-L_{i}(f) / \rho_{i}$. We have the following 
THEOREM 1.1. A is a self-adjoint operator on $\mathscr{H}$.

Proof. Since a set of $C_{0}^{\infty}\left(O_{1}\right)$ functions which vanish in a neighbourhood of $\partial \Omega_{1} \cup \partial \Omega_{2}$ is a dense subset of $\mathscr{H}, D_{N}$ is clearly a dense subset of $\mathscr{H}$. If $f$ belongs to $D_{N}$, from (1.6) we have that

$$
(A f, g)_{\mathscr{H}}=\sum_{i=1}^{i} \int_{\Omega_{i}}\left\{\sum_{i=1}\left(\lambda_{i} \delta_{j k}+2 \mu_{i}\right) \varepsilon_{j k}(f) \varepsilon_{j k}(\bar{g})\right\} d x
$$

for any $g \in\left(H^{1}\left(O_{1}\right)\right)^{3}$. From the above equality and the assumption $3 \lambda_{i}+$ $2 \mu_{i}>0$ it follows that $A \subset A^{*}$ and $A \geqq 0$. Thus in order to prove selfadjointness of $A$ we have to show that the range of $I+A$ is equal to $\mathscr{H}$. Let $\mathscr{H}^{1}\left(O_{1}\right)=\left(H^{1}\left(O_{1}\right)\right)^{3}$ be a Hilbert space with the inner product $(f, g)_{1}$ defined by $(f, g)_{\mathscr{H}}$ plus the right hand side of (1.7). By Korn's inequality on $O_{1}$ (see p. 110 of [1]) it follows that $\|f\|_{H^{1}\left(O_{1}\right)}^{2} \leqq C(f, f)_{1}$. This fact and Riesz's theorem imply that for any $g \in \mathscr{H}$ there exists $f \in \mathscr{H}^{1}\left(O_{1}\right)$ such that $(g, v)_{\mathscr{H}}=(f, v)_{1}$ for any $v \in H^{1}\left(O_{1}\right)$. From (1.6) this implies that $f \in D_{N}$ and $(I+A) f=g$. The proof is completed.

Since the domain $D(A)$ of $A$ is a dense subset of $\mathscr{H}^{1}\left(O_{1}\right)$ and the domain $D\left(A^{1 / 2}\right)$ of $A^{1 / 2}$ with the graph norm, we have the following

Proposition 1.2. The domain of $A^{1 / 2}$ is equal to $\mathscr{H}^{1}\left(O_{1}\right)$ and

$$
\left\|A^{1 / 2} f\right\|^{2}=\sum_{i=1}^{2}\left(\sum_{j, k}\left(\lambda_{i} \delta_{j k}+2 \mu_{i}\right)\left\|\varepsilon_{j k}(f)\right\|_{L^{2}\left(\Omega_{i}\right)}^{2}\right) .
$$

In (1.5) we assume that $f=\left(f_{1}, f_{2}\right) \in D_{N}$ and $g=\left(g_{1}, g_{2}\right) \in \mathscr{H}^{1}\left(O_{1}\right)$, then $u^{(i)}(x, t)=\left.u(x, t)\right|_{s_{i}}$, where $u(x, t)=\left(\cos t A^{1 / 2}\right) f+A^{-1 / 2}\left(\sin t A^{1 / 2}\right) g$, satisfies (1.1) and (1.5) in the distribution sense. On (1.2) (1.3) and (1.4) we have the following

Proposition 1.3. We put $\Gamma_{0}=\Gamma \backslash\left(\bar{\Gamma}_{1} \cup \bar{\Gamma}_{2}\right)$. Then $\left.u_{i}(x, t)\right|_{\Gamma_{0} \times R} \in$ $C\left(R_{t}: H_{\mathrm{loc}}^{1 / 2}\left(\Gamma_{0}\right)\right),\left.\sum_{j} n_{j}(x) \sigma_{j k}^{(i)}\left(u_{i}\right)\right|_{\Gamma_{h} \times R} \in C\left(R_{t}: H_{\mathrm{loc}}^{-1 / 2}\left(\Gamma_{h}\right)\right)$ where $h=0, i(i=1,2)$, and these satisfy (1.2), (1.3) and (1.4).

Proof. Since $u_{i}(x, t)$ belongs to $C\left(R_{t}: H^{1}\left(\Omega_{i}\right)\right)$, it follows that $\left.u_{i}(x, t)\right|_{\Gamma_{0} \times R}$ $\in C\left(R_{t}: H_{\text {loc }}^{1 / 2}\left(\Gamma_{0}\right)\right)$ and $u_{1}(x, t)=u_{2}(x, t)$ on $\Gamma_{0} \times R$. Let $x_{0}$ be a point of $\Gamma_{0}$ and $U$ be an open neighbourhood of $x_{0}$ such that $U \subset O_{1}$ and that there exists a diffeomorphism $\kappa$ from $U$ to $\left\{y \in R^{3}:|y|<\delta\right\}$ which maps $U \cap \Omega_{1}$ to $\left\{y \in R^{3}:|y|<\delta, y_{3}>0\right\}$. For any $\phi(x) \in C_{0}^{\infty}(U)$ we put $v_{i}(y, t)=\left(\phi u_{i}\right)\left(\kappa^{-1}(y), t\right)$. Then from Theorem 4.3.1 of [2] we see that $v_{1}(y, t) \in C\left(R_{t}: H_{(2,-1)}\left(\bar{R}_{+}^{3}\right)\right)$, where $H_{(m, s)}\left(\bar{R}_{+}^{3}\right)$ is a function space denoted in Definition 2.5.1 of [2]. By 
the trace theorem (see Theorem 2.5.6) in [2] it follows that the trace of $\sum_{j} n_{j}(x) \sigma_{j k}^{(1)}\left(u_{1}\right)$ on $\Gamma_{0} \times R$ belongs to $C\left(R_{t}: H_{\mathrm{loc}}^{-1 / 2}\left(\Gamma_{0}\right)\right)$. Put $v_{1 \varepsilon}(y, t)=$ $\varepsilon^{-2} \int \rho\left(\left(y^{\prime}-z^{\prime}\right) / \varepsilon\right) v_{1}\left(z^{\prime}, y_{3}^{\prime}, t\right) d z^{\prime}$, where a non-negative function $\rho\left(y^{\prime}\right)$ belongs to $C_{0}^{\infty}\left(\left\{y^{\prime} \in R^{2}:\left|y^{\prime}\right|<1\right\}\right)$ and $\int \rho\left(y^{\prime}\right) d y^{\prime}=1$. Then using Theorem 2.5.4 of [2], we can easily prove that $v_{1 \varepsilon}(y, t) \in C\left(R_{t}: H_{(2,0)}\left(\bar{R}_{+}^{3}\right)\right)$, supp $v_{1 \varepsilon} \subset\left\{y \in R^{3}:|y|<\delta\right\}$ if $\varepsilon$ is sufficiently small, and $v_{1 \varepsilon}(y, t)$ converges to $v_{1}(y, t)$ in the topology of $C\left(R_{t}: H_{(2,-1)}\left(\bar{R}_{+}^{3}\right)\right)$. These facts imply that the divergence theorem is valid for $\left(\sum_{j}\left(\sigma_{j k}^{(1)}\left(u_{1}\right) v_{j}\right)\right)_{k=1,2,3}$, where $v={ }^{t}\left(v_{1}, v_{2}, v_{3}\right) \in\left(H^{1}\left(O_{1}\right)\right)^{3}$. Similarly we can prove the same fact for $\phi u_{2}$. By (1.6) it follows that

$$
\int_{\Gamma_{0}} \sum_{j, k} n_{j}(x)\left\{\sigma_{j k}^{(1)}\left(u_{1}\right)-\sigma_{j k}^{(2)}\left(u_{2}\right)\right\} v_{k}(x) d x=0
$$

for any $v(x) \in\left(C_{0}^{\infty}(U)\right)^{3}$. The (1.3) is valid. Similarly we can prove (1.4). The proof is completed.

Remark 1.4. The arguments used in this section are easily extended for finite number of media which are not isotropic, whose displacements satisfy the similar boundary value problem to (1.1) to (1.5).

\section{$\S 2$. Reduction to first order systems and definition of rays}

In this section in order to study propagation of singularities to the solutions of (1.1) to (1.3) we shall reduce the considered boundary value problem to the first order system. After that we shall define an incident rays, a reflected ray, a transferred reflected ray, a refracted ray and a transferred refracted ray, which are half null bicharacteristics of $\tau^{2}-\alpha_{j}^{2}|\xi|^{2}$ or $\tau^{2}-\beta_{j}^{2}-\beta_{\jmath}^{2}|\xi|^{2}$.

Let us consider a solution $u_{i}(x, t)$ of (1.1). Hereafter we assume that $u_{i}$ is an extensible distribution, that is, there exists a distribution $U_{i}(x, t)$ on $R^{4}$ such that $U_{i}=u_{i}$ on $\Omega_{i} \times R$. Thus by Theorem 4.3 .1 of [2] the traces of $\left.u_{i}\right|_{\Gamma_{0} \times R}$ and $\left.\sigma_{j k}^{(i)}\left(u_{i}\right)\right|_{\Gamma_{0} \times R}$ are distributions on $\Gamma_{0} \times R$, and we can suppose these distributions satisfy the conditions (1.2) and (1.3). From now on we assume that $n(x)$ appearing in (1.3) is the unit outer normal vector of $\Omega_{1}$ at $\Gamma_{0}$. Since the boundary value problem (1.1) to (1.3) is rotation free, we may assume that the origin of $R^{3}$ belongs to $\Gamma_{0}$ and $n(0)={ }^{t}(0,0,-1)$. In a neighbourhood $U_{0}$ of $0 \Gamma_{0}$ is defined by $x_{3}=g\left(x^{\prime}\right)$, where $x^{\prime}=\left(x_{1}, x_{2}\right)$. Making use of the coordinate transform $\kappa ; y^{\prime}=x^{\prime}$, $y_{3}=x_{3}-g\left(x^{\prime}\right)$ such that $\Omega_{1} \cap U_{0}$ is tranformed into $\left\{y: y_{3}>0\right\}$ and putting 
$U_{i}(y, t)={ }^{t}\left(\Lambda\left(D_{y^{\prime}}, D_{t}\right)^{t} u_{i}, D_{y_{3}}{ }^{t} u_{i}\right)$, where $\Lambda$ is a pseudo-differential operator with the symbol $\Lambda_{1}\left(\eta^{\prime}, \tau\right)=\left(\left|\eta^{\prime}\right|^{2}+\tau^{2}+1\right)^{1 / 2}$, the problem (1.1) to (1.3) is reduced to the following boundary value problem (1.1) to (1.3) is reduced to the following boundary value problem (see section 1.1 of [9])

$$
\begin{cases}D_{y_{3}} U_{i}=M_{i}\left(y^{\prime}, D_{y^{\prime}}, D_{t}\right) U_{i} & \text { in }(-1)^{i+1} y_{3}>0 \\ \left(I_{3}, 0\right) U_{1}=\left(I_{3}, 0\right) U_{2} & \text { on } y_{3}=0 \\ B_{1}\left(y^{\prime}, D_{y^{\prime}}, D_{t}\right) U_{1}=B_{2}\left(y^{\prime}, D_{y^{\prime}}, D_{t}\right) U_{2} & \text { on } y_{3}=0\end{cases}
$$

where $I_{3}$ is the $3 \times 3$ identity matrix and the principal symbol $\left(B_{i 1}, B_{i 2}\right)$ $\left(y^{\prime}, \eta^{\prime}, \tau\right)$ of $B_{i}=\left(B_{i 1}, B_{i 2}\right)\left(y^{\prime}, D_{y^{\prime}}, D_{t}\right)$ is

$$
\left\{\begin{array}{l}
B_{i 1}\left(y^{\prime}, \eta^{\prime}, \tau\right)=\left(\lambda_{i} G^{t} \bar{\eta}+\mu_{i} \bar{\eta}^{t} G+\mu_{i} G \cdot \bar{\eta}\right) \Lambda_{1}^{-1}, \\
B_{i 2}\left(y^{\prime}, \eta^{\prime}, \tau\right)=\left(\lambda_{i}+\mu_{i}\right) G^{t} G+\mu_{i}|G|^{2} I_{3}
\end{array}\right.
$$

with $G={ }^{t}\left(-\nabla g\left(y^{\prime}\right), 1\right)$ and $\bar{\eta}={ }^{t}\left(\eta_{1}, \eta_{2}, 0\right)$. Here the principal symbol $M_{i 1}\left(y^{\prime}, \eta^{\prime}, \tau\right)$ of $M_{i 1}\left(y^{\prime}, D_{y^{\prime}}, D_{t}\right)$ satisfies that $\operatorname{det}\left(\eta_{3} I_{6}-M_{i 1}\right)=\left(\left(\eta_{3}-a\right)^{2}+\right.$ $\left.p_{i}\right)\left(\left(\eta_{3}-a\right)^{2}+s_{i}\right)^{2}$, where $a\left(y^{\prime}, \eta^{\prime}\right)=\eta^{\prime} \cdot \nabla g\left(y^{\prime}\right) /|G|^{2}, s_{i}\left(y^{\prime}, \eta^{\prime}, \tau\right)=\left(\left|\eta^{\prime}\right|^{2}-\tau^{2} / \alpha_{i}^{2}\right.$ $\left.-\left(\eta^{\prime} \cdot \nabla g\right) /|G|^{2}\right) /|G|^{2}$ and $p_{i}\left(y^{\prime}, \eta^{\prime}, \tau\right)=\left(\left|\eta^{\prime}\right|^{2}-\tau^{2} / \beta_{i}^{2}-\left(\eta^{\prime} \cdot \nabla g\right) /|G|^{2}\right) /|G|^{2}$ with $\alpha_{i}^{2}=\mu_{i} / \rho_{i}$ and $\beta_{i}^{2}=\left(\lambda_{i}+2 \mu_{i}\right) / \rho_{i}$.

We shall use notions of wave front set $W F(G)$ for $G\left(y^{\prime}, t\right) \in \mathscr{D}^{\prime}\left(R_{y^{\prime}, t}^{3}\right)$ defined in [3] and micro-local smoothness of $F \in C^{\infty}\left([0, \delta]: \mathscr{D}^{\prime}\left(R_{y^{\prime}, t}^{3}\right)\right) \cup$ $C^{\infty}\left([-\delta, 0]: \mathscr{D}^{\prime}\left(R_{y^{\prime}, t}^{3}\right)\right)$ at $\rho \in T^{*}\left(R_{y^{\prime}, t}^{3}\right) \backslash 0$, which means that there exists a properly supported pseudo-differential operator $A\left(y^{\prime}, t, D_{y^{\prime}}, D_{t}\right)$ such that $A$ is elliptic at $\rho$ and $(A F)(y, t) \in C^{\infty}\left([0, \pm \varepsilon] \times R_{y^{\prime}, t}^{3}\right)$ for some $\varepsilon>0$.

Let us consider a point $\left(0, \eta_{0}^{\prime}, \tau_{0}\right)$ such that $\tau_{0}\left|\eta_{0}\right|\left(p_{1} p_{2} s_{1} s_{2}\right)\left(0, \eta_{0}^{\prime}, \tau_{0}\right) \neq 0$. Put $\tilde{a}_{i}^{ \pm}\left(y^{\prime}, \eta^{\prime}, \tau\right)=a \pm(-1)^{i+1}\left(-s_{i}\right)^{1 / 2}$ if $s_{i}\left(0, \eta_{0}^{\prime}, \tau_{0}\right)>0$ and put $\tilde{a}_{i}^{ \pm}\left(y^{\prime}, \eta^{\prime}, \tau\right)$ $=a \pm(-1)^{i+1} \varepsilon\left(-s_{i}\right)^{1 / 2}$ if $s_{i}\left(0, \eta_{0}^{\prime}, \tau_{0}\right)<0$ and $\varepsilon=\operatorname{sgn} \tau_{0}$, where the branch of $\left(-s_{i}\right)^{1 / 2}$ is taken like that $(-1)^{1 / 2}$ is the imaginary unit. Similarly making use of $p_{i}$ instead of $s_{i}$, we define $\tilde{b}_{i}^{ \pm}\left(y^{\prime}, \eta^{\prime}, \tau\right)$. Then by Lemma 1.1 of [9] $\tilde{a}_{i}^{ \pm}$and $\tilde{b}_{i}^{ \pm}$are eigen values of $M_{i 1}\left(y^{\prime}, \eta^{\prime}, \tau\right)$ and there exist eigen vectors $s_{i k}^{ \pm}(k=1,2)$ and $s_{i 3}^{ \pm}$of $\tilde{a}_{i}^{ \pm}$and $\tilde{b}_{i}^{ \pm}$, respectively, which are linearly independent. By the argument of Sections 1.2 and 1.3 of [9] (see also Section 2 of [8]) we can reduce the boundary value problem (1.2) as follows: There exists an elliptic pseudo-differential operator $S_{i}\left(y^{\prime}, D_{y^{\prime}}, D_{t}\right)$ of order 0 defined in a conic neighbourhood of $\rho_{0}=\left(0, t_{0}, \eta_{0}^{\prime}, \tau_{0}\right) \in T^{*}\left(R_{y^{\prime}, t}^{3}\right) \backslash 0$ with the principal symbol $\left(s_{11}^{+}, s_{12}^{+}, s_{13}^{+}, s_{11}^{-}, s_{12}^{-}, s_{13}^{-}\right)$such that the boundary value problem (2.1) is micro-locally reduced to the following 


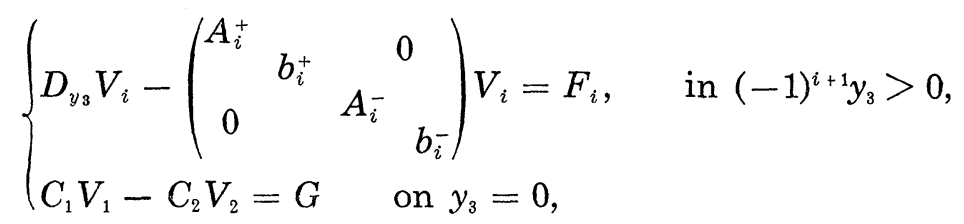

where $V_{i}=S_{i}^{-1} U_{i}, C_{i}\left(y^{\prime}, D_{y^{\prime}}, D_{t}\right)=\left(\begin{array}{ll}I_{3} & 0 \\ B_{i 1} & B_{i 2}\end{array}\right) S_{i}, \quad \rho_{0} \notin W F(G)$ and $F_{i}$ is smooth at $\rho$. Moreover the principal symbol of $A_{i}^{ \pm}\left(y^{\prime}, S_{y^{\prime}}, D_{t}\right)$ is the diagonal matrix $\tilde{a}_{i}^{ \pm}\left(y^{\prime}, \eta^{\prime}, \tau\right) I_{2}$ and the principal symbol of $b_{i}^{ \pm}\left(y^{\prime}, D_{y^{\prime}}, D_{t}\right)$ is $\tilde{b}_{i}^{ \pm}\left(y^{\prime}, \eta^{\prime}, \tau\right)$. At $y=0$ the principal symbols of $C_{1}$ and $C_{2}$ are simplified as follows:

LEMMA 2.1. If $\tau_{0}\left|\eta_{0}^{\prime}\right|\left(p_{1} p_{2} s_{1} s_{2}\right)\left(0, \eta_{0}^{\prime}, \tau_{0}\right) \neq 0$, then we may assume that at $y^{\prime}=0$ the principal symbol $C_{i}=\left(C_{i}^{+}, C_{i}^{-}\right)$of $C_{i}\left(y^{\prime}, D_{y^{\prime}}, D_{t}\right)$ is given by $C_{\imath}^{ \pm}={ }^{t}\left({ }^{t} C_{i 1}^{ \pm},{ }^{t} C_{i 2}^{ \pm}\right)$, where

$$
\begin{gathered}
C_{i 1}^{ \pm}=\left(\begin{array}{ccc}
a_{i}^{ \pm}\left|\eta^{\prime}\right|^{2} \Lambda_{1}^{-3} & 0 & \left|\eta^{\prime}\right|^{2} \Lambda_{1}^{-2} \\
0 & \left|\eta^{\prime}\right|^{2} \Lambda_{1}^{-2} & 0 \\
-\left|\eta^{\prime}\right|^{2} \Lambda_{1}^{-2} & 0 & b_{i}^{ \pm} \Lambda_{1}^{-1}
\end{array}\right), \\
C_{i 2}^{ \pm}=\left(\begin{array}{ccc}
\left(\rho_{i} \tau^{2}-2 \mu_{i}\left|\eta^{\prime}\right|^{2}\right)\left|\eta^{\prime}\right|^{2} \Lambda_{1}^{-4} & 0 & 2 \mu_{i} b_{i}^{ \pm}\left|\eta^{\prime}\right|^{2} \Lambda_{1}^{-3} \\
0 & \mu_{i} a_{i}^{ \pm}\left|\eta^{\prime}\right|^{2} \Lambda_{1}^{-3} & 0 \\
-2 \mu_{i} a_{i}^{ \pm}\left|\eta^{\prime}\right|^{2} \Lambda_{1}^{-3} & 0 & \left(\rho_{i} \tau^{2}-2 \mu_{i}\left|\eta^{\prime}\right|^{2}\right) \Lambda_{1}^{-2}
\end{array}\right)
\end{gathered}
$$

with $a_{i}^{ \pm}\left(\eta^{\prime}, \tau\right)=\tilde{a}_{i}^{ \pm}\left(0, \eta^{\prime}, \tau\right)$ and $b_{\imath}^{ \pm}(\eta, \tau)=\tilde{b}_{i}^{ \pm}\left(0, \eta^{\prime}, \tau\right)$.

Proof. In order to simplify the principal symbol of $C_{i}\left(y^{\prime}, D_{y^{\prime}}, D_{t}\right)$ we use an elliptic pseudo-differential operator $D_{0}\left(D_{y^{\prime}}, D_{t}\right)=\left(\begin{array}{cc}D & 0 \\ 0 & D\end{array}\right)$ of order 0 , where the principal symbol $d_{i j}\left(\eta^{\prime}, \tau\right)$ of the components of $3 \times 3$ matrix $D\left(D_{y^{\prime}}, D_{t}\right)$ are $d_{11}=d_{22}=\eta_{1} \Lambda_{1}^{-1}\left(\eta^{\prime}, \tau\right), d_{12}=-d_{21}=\eta_{2} \Lambda_{1}^{-1}\left(\eta^{\prime}, \tau\right), d_{33}=1$ and $d_{13}=d_{23}=d_{31}=d_{32}=0$. By (1.8) of [9] we can take the principal symbol of $S_{i}$ as follows; $s_{i 1}^{ \pm}={ }^{t}\left({ }^{t} w_{i 1}^{ \pm}, \tilde{a}_{i}^{ \pm} \Lambda_{1}^{-1}{ }^{t} w_{i 1}\right)$ with ${ }^{t} w_{i 1}^{ \pm}=\left(\tilde{a}_{i}^{ \pm}\left(\eta^{\prime}-\tilde{a}_{i}^{ \pm} \nabla g\right),-\mid \eta^{\prime}-\right.$ $\left.\left.\tilde{a}_{i}^{ \pm} \nabla g\right|^{2}\right) \Lambda_{1}^{-2}, s_{\imath 2}^{ \pm}={ }^{t}\left(w_{\imath 2}^{ \pm}, \tilde{a}_{i}^{ \pm} \Lambda_{1}^{-1 t} w_{\imath 2}^{ \pm}\right)$with ${ }^{t} w_{i 2}^{ \pm}=\left(-\left(\eta_{2}-\tilde{a}_{\imath}^{ \pm} \partial g / \partial y_{2}\right), \eta_{1}-\tilde{a}_{i}^{ \pm} \partial g / \partial y_{1}\right.$, 0) $\Lambda_{1}^{-1}$ and $s_{i 3}^{ \pm}={ }^{t}\left({ }^{t} w_{i 3}^{ \pm}, \tilde{b}_{i}^{ \pm} \Lambda_{1}^{-1} w_{i 3}^{ \pm}\right)$with ${ }^{t} w_{13}^{ \pm}=\left(\eta^{\prime}-b_{i} \nabla g, b_{i}^{ \pm}\right) \Lambda_{1}^{-1}$. Since $\nabla g=0$ at $y^{\prime}=0$, making use of (2.2), we can compute the principal symbol $D_{0} C_{i}\left(y^{\prime}, D_{y^{\prime}}, D_{t}\right)$, which is given by (2.4) at $y^{\prime}=0$. The proof is completed.

Let us consider the incident $P$ ray $P_{i}(\omega)$ in $\Omega_{1}$ hitting on $\left(0, t_{0}\right)$ with a direction $\omega=\left(\omega_{1}, \omega_{2}, \omega_{3}\right) \in S^{2}$ such that $0<n(0) \cdot \omega<1$, which is the half connected null bicharacteristic $\left\{\left(-\beta^{\top} \omega t, t_{0}-t,-\varepsilon \omega, \varepsilon \beta_{1}\right) \in T^{*}\left(\Omega_{1} \times R\right): t>0\right\}$ of $\tau^{2}-\beta_{1}^{2}|\xi|^{2}$ passing through $\rho_{0}=\left(0, t_{0},-\varepsilon \omega, \varepsilon \beta_{1}\right)$, where $\varepsilon^{2}=1$. Since 
the outer unit normal vector of $\Omega_{1}$ at 0 is $(0,0,-1)$, the reflected $P$ ray $P_{r}(\omega)$ of $P_{i}(\omega)$ is given by the connected ray $\left\{\left(\beta_{1} \omega_{r} t, t+t_{0},-\varepsilon \omega_{r}, \varepsilon \beta_{1}\right) \in\right.$ $\left.T^{*}\left(\Omega_{1} \times R\right): t>0\right\}$, where $\omega_{r}=\left(\omega_{1}, \omega_{2},-\omega_{3}\right)$. Hereafter we say that a ray $\gamma(t)$ in $\Omega_{i}$ parametrized by time $t$ is outgoing (incoming) if $(-1)^{i}\left(d x_{3} / d t\right)(0)$ $<0(>0)$, where $x_{3}(t)$ is the $x_{3}$ component of $\gamma(t)$. The half connected outgoing null bicharacteristic $S_{\mathrm{tr}}(\omega)$ of $\tau^{2}-\alpha_{1}^{2}|\xi|^{2}$ in $T^{*}\left(\Omega_{1} \times R\right)$ passing through $\rho$ with $\pi(\rho)=\pi\left(\rho_{0}\right)$ is called the transferred reflected ray of $P_{i}(\omega)$, where $\pi$ is the projection from $T^{*}\left(R_{x, t}^{4}\right)$ to $T^{*}(\partial \Omega \times R)$. If there exists the half connected outgoing null bicharacteristic $\tilde{P}_{r}(\omega)\left(\tilde{S}_{\mathrm{tr}}(\omega)\right)$ of $\tau^{2}-\beta_{2}^{2}|\xi|^{2}$ $\left(\tau^{2}-\alpha_{2}^{2}|\xi|^{2}\right)$ in $T^{*}\left(\Omega_{2} \times R\right)$ passing through with $\pi(\rho)=\pi\left(\rho_{0}\right)$, we call it the refracted $P$ ray (the transferred refracted $S$ ray) of $P_{i}(\omega)$. Similarly for the incident $S$ ray $S_{i}(\omega)$ passing through $\rho_{1}=\left(0, t_{0},-\varepsilon \omega, \varepsilon \alpha_{1}\right)$, the reflected $S$ ray $S_{r}(\omega)$, the transferred reflected $P$ ray $P_{\mathrm{tr}}(\omega)$, the refracted $S$ ray $\tilde{S}_{r}(\omega)$ and the transferred refracted $P$ ray $\tilde{P}_{\mathrm{tr}}(\omega)$ are defined, if these rays exist. The rays are concretely denoted as follows:

Lemma 2.2. i) Put $\omega_{\mathrm{tr}}^{ \pm}=\left(\omega^{\prime}, \pm\left(\beta_{1}^{2} / \alpha_{1}^{2}-1+(n(0) \cdot \omega)^{2}\right)^{1 / 2}\right)$, then $S_{\mathrm{tr}}(\omega)=$ $\left\{\left(\alpha_{1}^{2} \omega_{\mathrm{tr}}^{+} t / \beta_{1}, t+t_{0},-\varepsilon \omega_{\mathrm{tr}}^{+}, \varepsilon \beta_{1}\right) \in T^{*}\left(\Omega_{1} \times R\right): t>0\right\} . A$ similar statement is valid for $P_{\mathrm{tr}}(\omega)$, if $1-\alpha_{1}^{2} / \beta_{1}^{2}<(n(0) \cdot \omega)^{2}$.

ii) Put $\tilde{\omega}_{r}^{ \pm}=\left(\omega^{\prime}, \pm\left(\beta_{1}^{2} / \beta_{z}^{2}-1+(n(0) \cdot \omega)^{2}\right)^{1 / 2}\right)$, if $1-\beta_{1}^{2} / \beta_{2}^{2}<(n(0) \cdot \omega)^{2}$; then $\tilde{P}_{r}(\omega)=\left\{\left(\beta_{2}^{2} \tilde{\omega}_{r}^{-} t / \beta_{1}, t+t_{0},-\varepsilon \tilde{\omega}_{r}^{-}, \varepsilon \beta_{1}\right) \in T^{*}\left(\Omega_{2} \times R\right): t>0\right\}$. Similarly $\tilde{S}_{r}(\omega)$ is defined, if $1-\alpha_{1}^{2} / \alpha_{2}^{2}<(n(0) \cdot \omega)^{2}$.

iii) Put $\tilde{\omega}_{\mathrm{tr}}^{ \pm}=\left(\omega^{\prime}, \pm\left(\beta_{1}^{2} / \alpha_{2}^{2}-1+(n(0) \cdot \omega)^{2}\right)^{1 / 2}\right)$, if $1-\beta_{1}^{2} / \alpha_{2}^{2}<(n(0) \cdot \omega)^{2}$, then $\tilde{S}_{\mathrm{tr}}(\omega)=\left\{\left(\alpha_{2}^{2} \tilde{\omega}_{\mathrm{tr}}^{-} t / \beta_{1}, t+t_{0},-\varepsilon \tilde{\omega}_{\mathrm{tr}}^{-}, \varepsilon \beta_{1}\right) \in T^{*}\left(\Omega_{2} \times R\right): t>0\right\}$. Similarly $\tilde{P}_{\mathrm{tr}}(\omega)$ is defined, if $1-\alpha_{1}^{2} / \beta_{2}^{2}<(n(0) \cdot \omega)^{2}$.

The proof of Lemma 2.2 is easily derived from the definitions of the rays. For the incident $P$ ray $P_{i}(\omega)$ we also denote by $S_{\text {in }}(\omega), \tilde{P}_{\text {in }}(\omega)$ and $\tilde{S}_{\text {in }}(\omega)$ half connected incoming null bicharacteristics of $\tau^{2}-\alpha_{1}^{2}|u|^{2}, \tau^{2}-$ $\alpha_{2}^{2}|\xi|$ and $\tau^{2}-\beta_{2}^{2}|\xi|^{2}$ passing through $\left(0, t_{0},-\varepsilon \omega_{\mathrm{tr}}^{-}, \varepsilon \beta_{1}\right),\left(0, t_{0},-\varepsilon \tilde{\omega}_{r}^{+}, \varepsilon \beta_{1}\right)$ and $\left(0, t_{0},-\varepsilon \tilde{\omega}_{\mathrm{tr}}^{+}, \varepsilon \beta_{1}\right)$, repsectively, if these rays exist. Similarly for the incident $S$ ray $S_{i}(\omega) P_{\text {in }}(\omega), \tilde{P}_{\text {in }}(\omega)$ and $\tilde{S}_{\text {in }}(\omega)$ are defined.

\section{§3. Singularities corresponding to Stonly waves}

In this section we analyze singularities to a solution of (1.1) to (1.3) near an elliptic point $\left(0, t_{0}, \eta_{0}^{\prime}, \tau_{0}\right)$, that is, $s_{i}\left(0, \eta_{0}^{\prime}, \tau_{0}\right)>0$ for $i=1,2$. In [7] he proved that if $\Gamma_{0}$ is a hyperplane of $R^{3}$, there are surface waves satisfying (1.1) to (1.3) and propagating on the boundary $\Gamma_{0} \times R$. In this 
section without assuming the flatness of $\Gamma_{0}$, we shall show that there exist rays belonging to the wave front set of a solution of (1.1) to (1.3) which propagates in the elliptic region of the boundary $\Gamma_{0} \times R$.

We shall consider the Lopatinski matrix of the boundary value problem (2.3) in the elliptic region $\left\{\left(y^{\prime}, t, \eta^{\prime}, \tau\right): s_{i}\left(y^{\prime}, \eta^{\prime}, \tau\right)>0 i=1,2\right\}$, where $p_{i}\left(y^{\prime}, \eta^{\prime}, \tau\right)>0(i=1,2)$. We remark that if $y^{\prime}=0$, then the elliptic region is $\left\{\left(0, t, \eta^{\prime}, \tau\right): \min \left(\alpha_{1}^{2}, \alpha_{2}^{2}\right)\left|\eta^{\prime}\right|^{2}>\tau^{2}\right\}$.

Lemma 3.1. We assume that $\tau_{0} \neq 0$ and $\min \left(\alpha_{1}^{2}, \alpha_{2}^{2}\right)\left|\eta_{0}^{\prime}\right|^{2}>\tau_{0}^{2}$. Then the necessary and sufficient condition that the determinant $\left(C_{1}^{+}, C_{2}^{+}\right)$is zero is given by $F\left(\tau^{2} /\left|\eta^{\prime}\right|^{2}\right)=0$, where

$$
\begin{aligned}
F(s)= & \left\{\left(\rho_{2} a_{1}^{+}(s)-\rho_{1} a_{2}^{+}(s)\right)\left(\rho_{2} b_{1}^{+}(s)-\rho_{1} b_{2}^{+}(s)\right)+\left(\rho_{1}-\rho_{2}\right) s^{2}\right. \\
& -4\left(\mu_{1}-\mu_{2}\right)\left\{\rho_{1}\left(a_{2}^{+} b_{2}^{+}\right)(s)-\rho_{2}\left(a_{1}^{+} b_{1}^{+}\right)(s)+\rho_{1}-\rho_{2}\right\} s \\
& +4\left(\mu_{1}-\mu_{2}\right)^{2}\left\{\left(a_{1}^{+} b_{1}^{+} a_{2}^{+} b_{2}^{+}\right)(s)+\left(a_{2}^{+} b_{2}^{+}\right)(s)+\left(a_{2}^{+} b_{2}^{+}\right)(s)+1\right\}
\end{aligned}
$$

with $a_{j}^{+}(s)=(-1)^{j+1} i\left(1-s / \alpha_{j}^{2}\right)^{1 / 2}$ and $b_{j}^{+}(s)=(-1)^{j+1} i\left(1-s / \beta_{j}^{2}\right)^{1 / 2}$.

Proof. By (2.4) the second and fifth column vectors of $\left(C_{1}^{+}, C_{2}^{+}\right)$are linearly independent to the other column vectors, and these two vectors are linearly independent. Thus the condition $\operatorname{det}\left(C_{1}^{+}, C_{2}^{+}\right)=0$ is equivalent to the condition $\operatorname{det} M_{1}=0$, where $M_{1}$ is the $4 \times 4$ square matrix generated by eliminating the second and fifth column and line vectors of $\left(C_{1}^{+},-C_{2}^{+}\right)$. By simple calculations we can show that $\operatorname{det} M_{1}=C\left|\eta^{\prime}\right|^{6} F\left(\tau^{2} /\left|\eta^{\prime}\right|^{2}\right)$, where $C$ is a non zero constant. The proof is completed.

If we assume the Wiechert condition in seismology, that is, $\alpha_{1}=\alpha_{2}$ and $\beta_{1}=\beta_{2}$, then we can get informations on the roots of $F(s)=0$.

LEMMA 3.2. We assume $\alpha_{1}=\alpha_{2}$ and $\beta_{1}=\beta_{2}$, and put $\alpha=\alpha_{i}$ and $\beta=\beta_{i}$. Then we have the following statements.

i) The roots of $F(s)=0$ in $\left(0, \alpha^{2}\right)$ are also the roots of $f(x)=0$ in $(0,1)$ where $x=s / \alpha^{2}$ and $f(x)=\gamma x^{4}-\{\gamma+1+8 \gamma M\} x^{3}+\{1+24 \gamma M+8 M$ $\left.+8 \gamma M^{2}-M^{2}-16 \gamma^{2} M^{2}\right\} x^{2}-8\left\{\gamma M^{2}-4 \gamma^{2} M^{2}+3 M+2 \gamma M\right\} x+16\left(M+\gamma^{2} M\right)$ with $\gamma=\alpha / \beta$ and $M=\left(\rho_{1}-\rho_{2}\right)^{2} /\left(\rho_{1}+\rho_{2}\right)^{2}$.

ii) $f(x)$ has at least one root in $(0,1)$.

iii) If all roots $f(x)=0$ in $(0,1)$ are simple, then the set $\left\{y^{\prime}, t, \eta^{\prime}, \tau\right)$ : $\left.\operatorname{det}\left(C_{1}^{+}, C_{2}^{+}\right)\left(y^{\prime}, \eta^{\prime}, \tau\right)=0\right\}$ is locally given by $\tau=h\left(y^{\prime}, \eta^{\prime}\right)$, where $h\left(y^{\prime}, \eta^{\prime}\right)$ is a positively homogeneous function of degree 1.

Proof. In $F(s)$ put $s / \alpha^{2}=x$, then $F(s)$ is equal to $\alpha^{4}\left[\left(\rho_{1}-\rho_{2}\right)^{2}\left\{x^{2}+\right.\right.$ 
$\left.4(1-x)(2-\gamma x)\}-\left\{\left(\rho_{1}+\rho_{2}\right)^{2} x^{2}+4\left(\rho_{1}-\rho_{2}\right)^{2}(2-x)\right\}(1-\gamma x)^{1 / 2}(1-x)^{1 / 2}\right]=$ $\alpha^{4}\left[g_{1}(x)-g_{2}(x)\right]$, where $g_{i}(x)$ is defined by the last equality. Since $g_{1}(x)$ and $g_{2}(x)$ are positive if $x \in(0,1)$, the condition $F(s)=0$ is equivalent to $g_{2}^{2}(x)-g_{1}^{2}(x)=\left(\rho_{1}+\rho_{2}\right)^{2} x^{2} f(x)=0$. The statement ii) is clear, for $f(0)=$ $16 M\left(1-\gamma^{2}\right)>0$ and $f(1)=\left(\rho_{1}+\rho_{2}\right)^{-4}\left\{g_{2}^{2}(1)-g_{1}^{2}(1)\right\}=-M^{2}<0$. The statement iii) is a consequence of the implicit function theorem. The proof is completed.

We shall check conditions that the polynomial $f(x)$ has only simple roots in $(0,1)$.

Remark 3.3. i) One of the equivalent conditions that a polynomial $f(x)$ has a double roots is that the discriminant of $f(x)$ is zero. In this case the discriminant of $\left(\rho_{1}+\rho_{2}\right)^{2} \beta^{2} f(x)$ is a polynomial with respect to $\rho_{1}, \rho_{2}, \alpha$ and $\beta$. Thus for almost everywhere $\left(\rho_{1}, \rho_{2}, \alpha, \beta\right)$ the equation $f(x)$ $=0$ has only simple roots.

ii) A simple condition of simplicity of the roots in $(0,1)$ of $f(x)=0$ is given as follows: If $f^{\prime \prime}(1) \geqq 0$ and $(\gamma+1+8 M) / 4 \gamma \geqq 1$, where $x=$ $(\gamma+1+8 M) / 4 \gamma$ is the symmetric axis of $f^{\prime \prime}(x)=0$, then $f^{\prime \prime}(x)>0$ in $[0,1]$. This condition implies that $f(x)=0$ has only one root in $(0,1)$. Thus if $3 \gamma+8 M(\gamma M+1) \geqq\left(16 \gamma^{2}+1\right) M^{2}+2$ and $8 \gamma M+1 \geqq 3 \gamma$, then $f(x)=0$ has only one simple root in $(0,1)$.

Let us consider the Lopatinski determinant of the boundary value problem (2.3) in a conic neighbourhood of $\rho_{0}=\left(0, t_{0}, \eta_{0}^{\prime}, 0\right)$.

LEMmA 3.4. If $\tau_{0}=0$, then the Lopatinski determinant of (2.3), that is, the determinat of the principal symbol $\left(C_{1}^{+}, C_{2}^{+}\right)$is not zero at $\rho_{0}$.

Proof. In the case $\tau_{0}=0$, in Section 1.2 of [9] $S_{k}\left(0, \eta_{0}^{\prime}, 0\right)(k=1,2)$ is given as follows: Put $S_{k}\left(0, \eta_{0}^{\prime}, 0\right)=\left(s_{k 1}^{-}, s_{k 2}^{+}, s_{k 3}^{+}, s_{k 1}^{-}, s_{k 2}^{-}, s_{k 3}^{-}\right)$, then

$$
\begin{aligned}
& { }^{t} s_{k 1}^{ \pm}=\left( \pm(-1)^{k}{ }^{t} \eta_{0}^{\prime}, \pm(-1)^{k}\left|\eta_{0}^{\prime}\right|,\left(3 \beta_{k}-\alpha_{k}\right){ }^{t} \eta_{0}^{\prime}, \pm(-1)^{k+1} i\left(\beta_{k}-3 \alpha_{k}\right)\left|\eta_{0}^{\prime}\right|\right) \Lambda_{1}^{-1}, \\
& { }^{t} s_{k 2}^{ \pm}=\left({ }^{t} w_{k 2}, \pm(-1)^{k+1} i^{t} w_{k 2}\right) \quad \text { with }{ }^{t} w_{k 2}=\left(-\eta_{02}, \eta_{01}, 0\right) \Lambda_{1}^{-1}, \quad \text { and } \\
& { }^{t} s_{k 3}^{ \pm}=\left({ }^{t} w_{k 3}, \pm(-1)^{k+1} i^{t} w_{k 3}\right) \quad \text { with }{ }^{t} w_{k 3}=\left({ }^{s} \eta_{0}^{\prime}, \pm i\left|\eta_{0}^{\prime}\right|\right) \Lambda_{1}^{-1} .
\end{aligned}
$$

Using $S_{k}\left(0, \eta_{0}^{\prime}, 0\right)$ and (2.2) we can easily compute $\operatorname{det}\left(C_{1}^{+}, C_{2}^{+}\right)\left(0, \eta_{0}^{\prime}, 0\right)$, which is equal to $C\left(\mu_{1}+\mu_{2}\right) \times\left\{\mu_{1}\left(\lambda_{1}+2 \mu_{1}\right)+\mu_{2}\left(\lambda_{1}+3 \mu_{1}\right)\right\}\left\{\mu_{2}\left(\lambda_{2}+2 \mu_{2}\right)+\right.$ $\left.\mu_{1}\left(\lambda_{2}+3 \mu_{2}\right)\right\} / \rho_{1} \rho_{2}$ with a non zero constant $C$. That is not zero. The proof is completed.

For the solution $u_{i}$ of (1.1) we define $W F_{b}\left(u_{i}\right) \subset\left(T^{*}\left(\Omega_{i} \times R\right) \backslash 0\right) \cup$ $\left(T^{*}\left(\Gamma_{0} \times R\right) \backslash 0\right)$ as follows: i) $\rho \in T^{*}\left(\Omega_{i} \times R\right)$ belongs to $W F_{b}\left(u_{i}\right)$, if $\rho$ belongs 
to $W F\left(\left.u_{i}\right|_{\Omega_{i}}\right)$, ii) $\rho \in T^{*}\left(\Gamma_{0} \times R\right)$ does not belong to $W F_{b}\left(u_{i}\right)$, if $u_{i}\left(\kappa^{-1}(y), t\right)$ has the property of micro-local smoothness at $\pi\left(\kappa^{*}(\rho)\right)$, where $\pi$ is the projection from $T^{*}\left(R_{y, t}^{4}\right)$ to $T^{*}\left(\left\{y_{3}=0\right\} \times R\right)$ and $\kappa^{*}$ is the diffeomorphism from $T^{*}\left(U_{0} \times R\right)$ to $T^{*}\left(R_{y, t}^{4}\right)$ induced from $\kappa$, that is,

$$
\kappa^{*}(x, t, \xi, \tau)=\left(x^{\prime}, x_{3}-g\left(x^{\prime}\right), t, \xi^{\prime}+(\nabla g)\left(x^{\prime}\right) \xi_{3}, \xi_{3}, \tau\right) .
$$

This definition of $W F_{b}\left(u_{i}\right)$ is invariant by the diffeomorphism $\kappa$ (see Proposition 1.2 in [6]). Let $\Sigma_{e}^{\prime}$ be an elliptic region on the boundary, i.e., $\Sigma_{e}^{\prime}=\left\{\left(y^{\prime}, t, \eta^{\prime}, \tau\right) \in T^{*}\left(R_{y^{\prime}, t}^{3}\right) \backslash 0: s_{i}\left(y^{\prime}, \eta^{\prime}, \tau\right)>0, i=1,2\right\}$, and $\Sigma_{0}^{\prime}$ be a subset of $\Sigma_{e}^{\prime}$ such that $\operatorname{det}\left(C_{1}^{+}, C_{2}^{+}\right)\left(y^{\prime}, \eta^{\prime}, \tau\right)=0$. Put $\Sigma_{e}=\left(\pi \circ \kappa^{*}\right)^{-1}\left(\Sigma_{e}^{\prime}\right)$ and $\Sigma_{0}=$ $\left(\pi \circ \kappa^{*}\right)^{-1}\left(\Sigma_{0}^{\prime}\right)$; Then we have the following

Theorem 3.5. We assume that $\alpha_{1}=\alpha_{2}$ and $\beta_{1}=\beta_{2}$ and that $f(x)$ of Lemma 3.2 has only simple roots in $(0,1)$. Then $W F_{b}\left(u_{i}\right) \cap \Sigma_{e} \subset \Sigma_{0}$, where $\Sigma_{0}$ is locally given by $\tau-h(x, \xi)$ with $C^{\infty}$ homogeneous function $h(x, \xi)$ on $T^{*}\left(\Gamma_{0}\right) 0$ of order 1 , and $W F_{b}\left(u_{1}\right) \cup W F_{b}\left(u_{2}\right)$ is invariant under the Hamilton vector field $H_{\tau-h}$ on $T^{*}\left(\Gamma_{0} \times R\right) 0$.

Proof. In (2.3) we denote $V_{i}={ }^{t}\left(V_{i}^{+},{ }^{t} V_{i}^{-}\right)$. Then since $V_{1}^{-}$and $V_{2}^{-}$ satisfy backward parabolic equations, $\rho_{0}$ does not belong to $W F\left(\left.V_{1}^{-}\right|_{y_{3}=0}\right) \cup$ $W F\left(\left.V_{2}^{-}\right|_{y_{3}=0}\right)$. It follows that $W F\left(\left.C_{1}^{+} V_{1}^{+}\right|_{y_{3}=0}-\left.C_{2}^{+} V_{2}^{+}\right|_{y_{3}=0}\right)$ does not contain $\rho_{0}$. By Hörmander's theorem on propagation of singularities (see Theorem 6.1 .1 of [4] we have the desired statement.

Remark 3.6. i) Let $\left\{f_{i}, g_{i}\right\}$ be the initial data of the solution $u(x, t)$ of (1.1) to (1.5). Assume that $\left(x_{0}, \xi_{0}\right) \in W F\left(f_{i \mid \Gamma_{0}}\right) \cup W F\left(\left.g_{i}\right|_{\Gamma_{0}}\right)$, Then by Theorem 2.5.11' of [3] there exists $\tau_{0}$ such that an element $\rho_{0}=\left(x_{0}, 0, \xi_{0}, \tau_{0}\right)$ of $T^{*}\left(\Gamma_{0} \times R\right)$ belongs to $W F\left(\left.u\right|_{\Gamma_{0} \times R}\right)$. If $\rho_{0}$ is an elliptic point, then by Theorem 3.5 there exists a ray belonging to $W F_{b}\left(u_{1}\right) \cup W F_{b}\left(u_{1}\right)$, which starts at $\rho_{0}$ and propagates on the border $\Gamma_{0} \times R$.

ii) From the form of $F(s)$, the null points of $F(s)=0$ are roots of some polynomial of degree 22 whose coefficients are polynomial of $\left(\rho_{1}, \lambda_{1}, \mu_{1}, \rho_{2}, \lambda_{2}, \mu_{2}\right)$. Thus for almost all $\left(\rho_{1}, \lambda_{1}, \mu_{1}, \rho_{2}, \lambda_{2}, \mu_{2}\right)$ with $\left.(F(s) / s)^{2}\right|_{s=0}$ $F\left(\min \left(\alpha_{1}^{2}, \alpha_{2}^{2}\right)\right)<0$ Theorem 3.5 holds.

\section{§ 4. Incident $P$ singularities}

In the case that $\alpha_{2}<\beta_{1}<\beta_{2}$ and $\alpha_{1} \neq \alpha_{2}$ there exist interesting reflective and refractive phenomena. Thus in this section we assume the above condition. We shall consider incident $P$ singularities and show 
the following theorems on reflective and refractive phenomena of singularities.

THEOREM 4.1. i) We assume $\beta_{1}^{2}<\beta_{2}^{2}\left(1-(n(0) \cdot \omega)^{2}\right)$. Then $\tilde{P}_{\text {in }}(\omega)$ and $\tilde{P}_{r}(\omega)$ do not exist. Furthermore we suppose that $S_{\mathrm{in}}(\omega) \cap W F\left(u_{1}\right)=\tilde{S}_{\mathrm{in}}(w)$ $\cap W F\left(u_{2}\right)=\phi \quad$ and $\quad P_{i}(\omega) \subset W F\left(u_{1}\right)$. Then $P_{r}(\omega) \cup S_{\mathrm{tr}}(\omega) \subset W F\left(u_{1}\right)$ and $\tilde{S}_{\mathrm{tr}}(\omega) \subset W F\left(u_{2}\right)$.

ii) We assume $\beta_{1}^{2}>\beta_{2}^{2}\left(1-(n(0) \cdot \omega)^{2}\right)$. Then there exists a function $G_{1}(s)$ whose null points are at most 30 such that if $\left.G_{1}\left(\beta_{1}^{2} /(1-n(0) \cdot \omega)^{2}\right)\right) \neq 0$, $S_{\text {in }}(\omega) \cap W F\left(u_{1}\right)=\left(\tilde{P}_{\text {in }}(\omega) \cup \tilde{S}_{\text {in }}(\omega)\right) \cap W F\left(u_{2}\right)=\phi$ and $P_{i}(\omega) \subset W F\left(u_{1}\right)$, then $P_{r}(\omega) \cup S_{\mathrm{tr}}(\omega) \subset W F\left(u_{1}\right)$ and $\tilde{P}_{r}(\omega) \cup \tilde{S}_{\mathrm{tr}}(\omega) \subset W F\left(u_{2}\right)$.

The concrete form of $G_{1}(s)$ is given in Lemma 4.3. The idea of proving the above theorem is as follows: First we shall look for an elliptic pseudodifferential operator $A$ such that some components of $A C_{i}^{-}(i=1,2)$ vanish. After that making use of the assumptions of $W F\left(u_{i}\right)$, we shall check the conditions to the wave front sets of the components of $\left.V_{i}\right|_{y_{3}=0}$ $(i=1,2)$, which derive the statements of the theorem.

Let $\rho_{0}$ be $\left(0, t_{0},-\varepsilon \omega, \varepsilon \beta_{1}\right)$, where $\varepsilon^{2}=1,0<n(0) \cdot \omega<1$. Then the projected point $\rho_{1}$ of $\rho_{0}$ to $T^{*}(\partial \Omega \times R)$ is $\left(0, t_{0},-\varepsilon \omega^{\prime}, \varepsilon \beta_{1}\right)$. In a conic neighbourhood $\Gamma_{1}$ of $\rho_{1}$ in $T^{*}\left(R_{y^{\prime}, t}^{3}\right) \backslash 0$ we may assume that at $y=0$ the principal symbols of $A_{i}^{ \pm}, b_{i}^{ \pm}$in $(2.3)$ are $a_{1}^{ \pm}\left(\eta^{\prime}, \tau\right) I_{2}=\left( \pm \varepsilon\left(\tau^{2} / \alpha_{1}^{2}-\left|\eta^{\prime}\right|^{2}\right)^{1 / 2}\right) I_{2}$, $a_{2}^{ \pm}\left(\eta^{\prime}, \tau\right) I_{2}=\left(\mp \varepsilon\left(\tau^{2} / \alpha_{2}^{2}-\left|\eta^{\prime}\right|^{2}\right)^{1 / 2}\right) I_{2}, \quad b_{1}^{ \pm}\left(\eta^{\prime}, \tau\right)= \pm \varepsilon\left(\tau^{2} / \beta_{1}^{2}-\left|\eta^{\prime}\right|^{2}\right)^{1 / 2}$, and $b_{2}^{ \pm}\left(\eta^{\prime}, \tau\right)$ is $\mp i\left(\left|\eta^{\prime}\right|^{2}-\tau^{2} / \beta_{2}^{2}\right)^{1 / 2}$ if $\beta_{1}^{2}<\beta_{2}^{2}\left(1-(n(0) \cdot \omega)^{2}\right)$ and is $\pm \varepsilon\left(\tau^{2} / \beta_{2}^{2}-\left|\eta^{\prime}\right|^{2}\right)^{1 / 2}$ if $\beta_{1}^{2}>$ $\beta_{2}^{2}\left(1-(n(0) \cdot \omega)^{2}\right)$. The boundary operator $C_{i}\left(y^{\prime}, D_{y^{\prime}}, D_{t}\right)$ of $(2.3)$ is also defined by using these notations. We say that a pseudodifferential operator $P\left(y^{\prime}, t, D_{y^{\prime}}, D_{t}\right)$ belongs to $L^{-\infty}(\Gamma)$, where $\Gamma$ is a conic open set of $T^{*}\left(R_{y^{\prime}, t}^{3}\right)$, if the symbol of $P$ is rapidly decreasing with respect to $\left(\eta^{\prime}, \tau\right)$ in $\Gamma$. We have the following

Lemma 4.2. There exists a pseudo-differential operator $A\left(y^{\prime}, D_{y^{\prime}}, D_{t}\right)$ of order 0 defined in a conic neighbourhood $\Gamma_{1}$ of $\rho_{1}$ such that the principal symbol $A_{0}\left(y^{\prime}, \eta^{\prime}, \tau\right)$ of $A$ is the identity matrix $I_{6}$ at $y^{\prime}=0$, and that the $(1,2),(2,1),(2,3),(3,2),(4,2),(5,1),(5,3)$ and $(6,2)$ components of $\left(A C_{i}^{-}\right)\left(y^{\prime}, D_{y^{\prime}}, D_{t}\right)(i=1,2)$ are 0 modulo $L^{-\infty}\left(\Gamma_{1}\right)$.

Proof. Put $C_{1}^{-}\left(y^{\prime}, D_{y^{\prime}}, D_{t}\right)=\left(c_{1}, c_{2}, c_{3}\right)\left(y^{\prime}, D_{y^{\prime}}, D_{t}\right), \quad C_{2}^{-}\left(y^{\prime}, D_{y^{\prime}}, D_{t}\right)=$ $\left(c_{4}, c_{5}, c_{6}\right)\left(y^{\prime}, D_{y^{\prime}}, D_{t}\right)$ and denote by $a_{j}\left(y^{\prime}, D_{y^{\prime}}, D_{t}\right)$ the $j$-th line vector of $A\left(y^{\prime}, D_{y^{\prime}}, D_{t}\right)$. Then the required conditions are 


$$
\begin{gathered}
a_{j} \cdot c_{2}=a_{j} \cdot c_{5}=0 \quad \text { modulo } L^{-\infty}\left(\Gamma_{1}\right)(j=1,3,4,6) \\
a_{j} \cdot c_{1}=a_{j} \cdot c_{3}=a_{j} \cdot c_{4}=a_{j} \cdot c_{6}=0 \quad \text { modulo } L^{-\infty}\left(\Gamma_{1}\right)(j=2,5) .
\end{gathered}
$$

We denote the symbol of $a_{j}$ by $\sum_{k=0}^{\infty} a_{j k}\left(y^{\prime}, \eta^{\prime}, \tau\right)$, where $a_{j k}$ is of order $-k$, and the principal symbol of $c_{j}$ by $c_{j 0}\left(y^{\prime}, \eta^{\prime}, \tau\right)$. Put $a_{10}=f_{1}+x_{12} c_{20}+x_{15} c_{50}$, where ${ }^{t} f_{1}=(1,0, \cdots, 0) \in R^{6}$. Then the condition $\left\langle a_{10}, c_{20}\right\rangle=\left\langle a_{10}, c_{50}\right\rangle=0$ is equivalent to

$$
\left(\begin{array}{ll}
\left\langle c_{20}, c_{20}\right\rangle & \left\langle c_{50}, c_{20}\right\rangle \\
\left\langle c_{20}, c_{50}\right\rangle & \left\langle c_{50}, c_{50}\right\rangle
\end{array}\right)\left(\begin{array}{l}
x_{12} \\
x_{15}
\end{array}\right)=-\left(\begin{array}{l}
\left\langle f_{1}, c_{20}\right\rangle \\
\left\langle f_{1}, c_{15}\right\rangle
\end{array}\right)
$$

Since from (2.4) $c_{20}$ and $c_{50}$ are linearly independent, we can solve the equation (4.3) and $x_{12}$ and $x_{15}$ are zero at $y^{\prime}=0$. Similarly if we put $a_{1 k}=x_{k 2} c_{20}+x_{k 5} c_{50}$, where $x_{k 2}\left(y^{\prime}, \eta^{\prime} \tau\right)$ and $x_{k 5}\left(y^{\prime}, \eta^{\prime}, \tau\right)$ are of order $-k$, we can decide the required $a_{1 k}\left(y^{\prime}, \eta^{\prime}, \tau\right)$. Similarly in order to construct $a_{j}\left(y^{\prime}, D_{y^{\prime}}, D_{t}\right)$ which satisfies (4.2) we only check that $c_{10}, c_{30}, c_{40}$ and $c_{60}$ are linearly independent. This condition is equivelent to the condition $\operatorname{det} M\left(\eta^{\prime}, \tau\right) \neq 0$, where $M\left(\eta^{\prime}, \tau\right)$ is the $4 \times 4$ square matrix which is generated by eliminating the second and fourth column and line vectors of $\left(C_{1}^{-}, C_{2}^{-}\right)\left(0, \eta^{\prime}, \tau\right)$. We have

$$
\begin{aligned}
& \operatorname{det} M\left(\eta^{\prime}, \tau\right)=\left[\left\{\rho_{2}^{2} a_{1}^{-} b_{1}^{-}+\rho_{1}^{2} a_{2}^{-} b_{2}^{-}-\rho_{1} \rho_{2}\left(a_{1}^{-} b_{2}^{-}+a_{2}^{-} b_{1}^{-}\right)+\left(\rho_{1}-\rho_{2}\right)^{2}\left|\eta^{\prime}\right|^{2}\right\} \tau^{4}\right. \\
& \quad+4\left(\mu_{1}-\mu_{2}\right)\left\{\rho_{2} a_{1}^{-} b_{1}^{-}-\rho_{1} a_{2}^{-} b_{2}^{-}-\left(\rho_{1}-\rho_{2}\right)\left|\eta^{\prime}\right|^{2}\right\}\left|\eta^{\prime}\right|^{2} \tau^{2} \\
& \left.\quad+4\left(\mu_{1}-\mu_{2}\right)^{2}\left\{a_{1}^{-} b_{1}^{-} a_{2}^{-} b_{2}^{-}+\left(a_{1}^{-} b_{1}^{-}+a_{2}^{-} b_{2}^{-}\right)\left|\eta^{\prime}\right|^{2}+\left|\eta^{\prime}\right|^{4}\right\}\left|\eta^{\prime}\right|^{2}\right]\left|\eta^{\prime}\right|^{4} \Lambda_{1}^{-10}
\end{aligned}
$$

If $b_{2}^{-}\left(\eta^{\prime}, \tau\right)$ is real valued, then $\left|\eta^{\prime}\right|^{-4} \Lambda_{1}^{10} \times(4.4)$ is equal to $\left\{\left(\rho_{1}-\rho_{2}\right) \tau^{2}-\right.$ $\left.2\left(\mu_{1}-\mu_{2}\right)\left|\eta^{\prime}\right|^{2}\right\}^{2}\left|\eta^{\prime}\right|^{2}+\left\{\rho_{2} \tau^{2}+2\left(\mu_{1}-\mu_{2}\right)\left|\eta^{\prime}\right|^{2}\right\}^{2} a_{1}^{-} b_{1}^{-}+\left\{\rho_{1} \tau^{2}-2\left(\mu_{1}-\mu_{2}\right)\left|\eta^{\prime}\right|^{2}\right\}^{2} a_{2}^{-} b_{2}^{-}$ $-\rho_{1} \rho_{2}\left(a_{1}^{-} b_{2}^{-}+a_{2}^{-} b_{1}^{-}\right) \tau^{4}+4\left(\mu_{1}-\mu_{2}\right)^{2} a_{1}^{-} b_{1}^{-} a_{2}^{-} b_{2}^{-}\left|\eta^{\prime}\right|^{2}$, which is positive because $a_{1}^{-} b_{1}^{-}$and $a_{2}^{-} b_{2}^{-}$are positive and $a_{1}^{-} b_{2}^{-}$and $a_{2}^{-} b_{1}^{-}$are negative. If $b_{2}^{-}\left(\eta^{\prime}, \tau\right)$ is pure imaginary, then $\operatorname{Re}\left(\operatorname{det} M\left(\eta^{\prime}, \tau\right)\right)$ is also positive. Thus $c_{10}, c_{30}, c_{40}$ and $c_{60}$ are linearly independent. The proof is completed.

Next we shall compute the principal symbol of $\left(C^{-}\right)^{-1} C_{1}^{+}$, where $C^{-}=\left(C_{1}^{-}, C_{2}^{-}\right)$, which is elliptic from the proof of Lemma 4.2, Define $a_{i}(s)=\left(s / \alpha_{i}^{2}-1\right)^{1 / 2}$ and $b_{i}(s)=\left(s / \beta_{i}^{2}-1\right)^{1 / 2}(i=1,2)$ and put

$$
\begin{gathered}
g_{1}(s)=\left(\rho_{2} s+2\left(\mu_{1}-\mu_{2}\right)\right)\left(\left(\rho_{2}-\rho_{1}\right) s+2\left(\mu_{1}-\mu_{2}\right)\right) \\
\quad-2\left(\mu_{1}-\mu_{2}\right)\left(\rho_{1} s-2\left(\mu_{1}-\mu_{2}\right)\right) a_{2}(s) b_{2}(s), \\
g_{3}(s)=\rho_{1} \rho_{2}\left(a_{1} b_{2}-b_{1} a_{2}\right)(s) s^{2}+\left(\rho_{2} s+2\left(\mu_{1}-\mu_{2}\right)\right)^{2}\left(a_{1} b_{1}\right)(s) \\
\quad-\left(\rho_{1} s-2\left(\mu_{1}-\mu_{2}\right)\right)^{2}\left(a_{2} b_{2}\right)(s)+4\left(\mu_{1}-\mu_{2}\right)^{2}\left(a_{1} b_{1} a_{2} b_{2}\right)(s) \\
-\left(\left(\rho_{1}-\rho_{2}\right) s-2\left(\mu_{1}-\mu_{2}\right)\right)^{2},
\end{gathered}
$$




$$
\begin{aligned}
g_{4}(s)=\{( & \left.\left.\rho_{1}-\rho_{2}\right)^{2}-4\left(\mu_{1}-\mu_{2}\right)^{2} /\left(\alpha_{1}^{2} \beta_{2}^{2}\right)\right\} s \\
& \quad-4\left(\mu_{1}-\mu_{2}\right)\left\{\left(\rho_{1}-\rho_{2}\right)-\left(\mu_{1}-\mu_{2}\right)\left(\alpha_{1}^{-2}+\beta_{2}^{-2}\right)\right\}, \\
g_{6}(s)= & \left(\rho_{2}^{1} / \alpha_{1}^{2}-\rho_{1}^{2} / \alpha_{2}^{2}\right) s^{2}+\left(\rho_{1}+\rho_{2}\right)\left\{\left(\rho_{1}-\rho_{2}\right)+4\left(\mu_{1}-\mu_{2}\right)\left(\alpha_{1}^{-2}+\alpha_{2}^{-2}\right)\right\} s \\
\quad & +4\left(\mu_{1}-\mu_{2}\right)\left\{\left(\mu_{1}-\mu_{2}\right)\left(\alpha_{1}^{-2}-\alpha_{2}^{-2}\right)-\left(\rho_{1}+\rho_{2}\right)\right\} .
\end{aligned}
$$

Then we have

LEMMA 4.3. i) If $\rho_{1} \neq \rho_{2}, \mu_{1} \neq \mu_{2}$ and $\beta_{1}^{2}<\beta_{2}^{2}\left(1-(n(0) \cdot \omega)^{2}\right)$, then the (1.3), (3.3) and (4.3) component of $\left(C^{-}\right)^{-1} C_{1}^{+}$are elliptic at $\rho_{1}$, where $C^{-}=$ $\left(C_{1}^{-},-C_{2}^{-}\right)$.

ii) If $\beta_{1}^{2}>\beta_{2}^{2}\left(1-\left(n(0 \cdot \omega)^{2}\right)\right.$ and $g_{j}\left(\beta_{1}^{2} /\left(1-(n(0) \cdot \omega)^{2}\right)\right) \neq 0$, then the $(j, 3)$ component of $\left(C^{-}\right)^{-1} C_{1}^{+}$is elliptic at $\rho_{1}$, where $j=1,3,4,6$.

iii) If $\alpha_{1} \neq \alpha_{2}$, then $G_{1}(s)=\left(g_{1} g_{3} g_{4} g_{6}\right)(s)$ has at most 30 null points in $\left(\beta_{1}^{2}, \infty\right)$.

Proof. Let us denote by $M_{1}\left(y^{\prime}, \eta^{\prime}, \tau\right)=\left(c_{1}, c_{2}, c_{3}, c_{4}\right)$ the $4 \times 4$ square matrix which is generated by eliminating the second and fourth column and line vectors of the principal symbol of $C^{-}\left(y^{\prime}, D_{y^{\prime}}, D_{t}\right)$ and put $c\left(y, \eta^{\prime}, \tau\right)$ to be the column vector which is generated by eliminating the second and fourth components of the principal symbol of the third column vector of $C_{1}^{+}\left(y^{\prime}, D_{y^{\prime}}, D_{t}\right)$. Then from Lemma 4.2 and Cramer's formula we may check that the determinant of $\left(c, c_{i_{1}}, c_{i_{2}}, c_{i_{3}}\right)$, where $1 \leqq i_{j} \leqq 4$ and $i_{j} \neq i_{k}$ if $j \neq k$, is not zero at $\rho_{1}$. From (2.4) it follows that

$$
\begin{aligned}
& \operatorname{det}\left(c, c_{2}, c_{3}, c_{4}\right)\left(0, \eta^{\prime}, \tau\right)=A_{1}\left[\left(\rho_{2} \tau^{2}+2\left(\mu_{1}-\mu_{2}\right)\left|\eta^{\prime}\right|^{2}\right)\right. \\
& \left.\quad \times\left(\left(\rho_{1}-\rho_{2}\right) \tau^{2}-2\left(\mu_{1}-\mu_{2}\right)\left|\eta^{\prime}\right|^{2}\right)+2\left(\mu_{1}-\mu_{2}\right)\left(\rho_{1} \tau^{2}-\left.2\left(\mu_{1}-\mu_{2}\right)\left|\eta^{\prime}\right|\right|^{2}\right) a_{2}^{-} b_{2}^{-}\right] \\
& \begin{aligned}
\operatorname{det}\left(c_{1}, c, c_{3}, c_{4}\right)\left(0, \eta^{\prime}, \tau\right)=A_{2}\left[\rho_{1} \rho_{2}\left(a_{1}^{-} b_{2}^{-}+a_{2}^{-} b_{1}^{+}\right) \tau^{4}\right. \\
\quad-\left(\rho_{2} \tau^{2}+2\left(\mu_{1}-\mu_{2}\right)\left|\eta^{\prime}\right|^{2}\right)^{2} a_{1}^{-} b_{1}^{+}-\left(\rho_{2} \tau^{2}-2\left(\mu_{1}-\mu_{2}\right)\left|\eta^{\prime}\right|^{2}\right)^{2} a_{2}^{-} b_{2}^{-} \\
\left.\quad-\left.\left(\left(\rho_{1}-p_{2}\right) \tau^{2}-2\left(\mu_{1}-\mu_{2}\right)\left|\eta^{\prime}\right|\right)^{2}\left|\eta^{\prime}\right|\right|^{2}\right] \\
\operatorname{det}\left(c_{1}, c_{2}, c, c_{4}\right)\left(0, \eta^{\prime}, \tau\right)=A_{3}\left\{\left(\rho_{1}-\rho_{2}\right) \tau^{2}-2\left(\mu_{1}-\mu_{2}\right)\left(\left|\eta^{\prime}\right|^{2}-a_{1}^{-} b_{2}^{-}\right)\right\} \\
\operatorname{det}\left(c_{1}, c_{2}, c_{3}, c\right)\left(0, \eta^{\prime}, \tau\right)=A_{4}\left\{\left(\rho_{2} \tau^{2}+2\left(\mu_{1}-\mu_{2}\right)\left|\eta^{\prime}\right|^{2}\right) a_{1}^{-}\right. \\
\left.-\left(\rho_{1} \tau^{2}-2\left(\mu_{1}-\mu_{2}\right)\left|\eta^{\prime}\right|^{2}\right) a_{2}^{-}\right\}
\end{aligned}
\end{aligned}
$$

where $A_{j}\left(\eta^{\prime}, \tau\right)$ is not zero, if $\eta^{\prime} \neq 0$ and $\tau \neq 0$. If $\rho_{1} \neq \rho_{2}, \mu_{1} \neq \mu_{2}$ and $b_{2}^{-}$ is pure imaginary, then the real part and the imaginary part of (4.5) do not vanish at the same point and the imaginary parts of (4.6) and (4.7) are not zero. It follows that the statement i) holds. (4.5) and (4.6) clearly implies that the statement ii) holds for $j=1,3, g_{4}(s)$ and $g_{6}(s)$ are equal 
to $\left[\left(\left(\rho_{1}-\rho_{2}\right) s-2\left(\mu_{1}-\mu_{2}\right)\right)^{2}-4\left(\mu_{1}-\mu_{2}\right)^{2}\left(a_{1}^{-} b_{2}^{-}\right)^{2}\right] / s$ and $\left[\left\{\left(\rho_{2} s+2\left(\mu_{1}-\mu_{2}\right)\right) a_{1}^{-}\right\}^{2}\right.$ $-\left\{\left(\rho_{1} s-2\left(\mu_{1}-\mu_{2}\right)\right) a_{2}^{-}\right\}^{2} / s$. Thus when $\rho_{1} \neq \rho_{2}$ and $\mu_{1} \neq \mu_{2}, g_{1}(s), g_{4}(s)$ and $g_{6}(s)$ are not identically zero and one of the equivalent conditions that $\xi_{1}(s)=0$ is $\left[\left\{\left(\rho_{2} s+2\left(\mu_{1}-\mu_{2}\right)\right)\left(\left(\rho_{1}-\rho_{2}\right) s+2\left(\mu_{1}-\mu_{2}\right)\right\}^{2}-4\left(\mu_{1}-\mu_{2}\right)^{2}\left\{\left(\rho_{1} s-\right.\right.\right.\right.$ $\left.\left.\left.-2\left(\mu_{1}-\mu_{2}\right)\right)\left(a_{2} b_{2}\right)(s)\right\}^{2}\right] / s=0$. It follows that the null points of $\left(g_{1} g_{4} g_{6}\right)(s)$ are at most 6. By the form of $g_{3}(s)$ the null points of $g_{3}(s)$ are roots of some polynomial of degree 24. These show that the null points of $G_{1}(s)$ in $\left(\beta_{1}^{2}, \infty\right)$ are at most 30 . The proof is completed.

The proof of Theorem 4.1. The proof of the statement i) is similar to that of the statement ii). Thus we only prove the statement ii). In (2.3) we put ${ }^{t} V_{i}={ }^{t}\left({ }^{t} V_{i}^{+},{ }^{t} V_{i}^{-}\right)$with $V_{i}^{ \pm}={ }^{t}\left(v_{i 1}^{+}, v_{i 2}^{ \pm}, v_{i 3}^{ \pm}\right)$. The assumption implies that $\rho_{1} \notin W F\left(\left.v_{i 1}^{+}\right|_{y_{3}=0}\right) \cup W F\left(\left.v_{i 2}^{+}\right|_{y_{3}=0}\right) \cup W F\left(\left.v_{2}^{+}\right|_{y_{3}=0}\right)$ and $\rho_{1} \in W F\left(\left.v_{i 3}^{+}\right|_{y_{3}=0}\right)$, where $\rho_{1}=\left(0, t_{0},-\varepsilon \omega^{\prime}, \varepsilon \beta_{1}\right)$. Thus the boundary condition in (2.3) is reduced to the following

$$
{ }^{t}\left({ }^{t} V_{1}^{-},{ }^{t} V_{2}^{-}\right)=-\left(\left(C^{-}\right)^{-1} c_{13}^{+}\right) v_{13}^{+}+\tilde{G},
$$

where $c_{13}^{+}\left(y^{\prime}, D_{y^{\prime}}, D_{t}\right)$ is the third column vector of $C_{1}^{+}\left(y^{\prime}, D_{y^{\prime}}, D_{t}\right)$ and $\rho_{1}$ does not belong to $W F(\tilde{G})$. From Lemma 4.3 and (4.9) we see the $\rho_{1} \in$ $W F\left(v_{11}^{-}\right) \cap W F\left(v_{13}^{-}\right) \cap W F\left(v_{21}^{-}\right) \cap W F\left(v_{22}^{-}\right)$. Using Theorem 2.5.11' of [3], we have the desired conclusions. The proof is completed.

\section{$\S 5$. Incident $S$ singularities}

Let us consider incident $S$ singularities. So all functions and pseudodifferential operators are defined in $\Gamma_{2}$, where $\Gamma_{2}$ is a conic neighbourhood of $\rho_{1}=\left(0, t_{0},-\varepsilon \omega^{\prime}, \varepsilon \alpha_{1}\right)$. Under the assumption that $\alpha_{2}<\beta_{1}<\beta_{2}$ and $\alpha_{1} \neq \alpha_{2}$ we shall show the following

TheOREM 5.1. We assume that $\beta_{1}^{2}<\alpha_{1}^{2} /\left(1-(n(0) \cdot \omega)^{2}\right)<\beta_{2}^{2}$. Then $\tilde{P}_{\text {in }}(\omega)$ and $\tilde{P}_{r}(\omega)$ do not exist and there exists a function $H_{1}(s)$ whose null points are at most 48 such that if $H_{1}\left(\alpha_{1}^{2} /\left(1-(n(0) \cdot \omega)^{2}\right)\right) \neq 0, S_{i}(\omega) \subset W F\left(u_{1}\right)$ and $P_{\mathrm{in}}(w) \cap W F\left(u_{1}\right)=\tilde{S}_{\mathrm{in}}(\omega) \cap W F\left(u_{2}\right)=\phi$, then one of the following two cases occurs; a) $S_{r}(\omega) \cup P_{\mathrm{tr}}(\omega) \subset W F\left(u_{1}\right)$ and $\tilde{S}_{r}(\omega) \subset W F\left(u_{2}\right)$, b) $S_{r}(\omega) \subset W F\left(u_{1}\right)$, $\tilde{S}_{r}(\omega) \subset W F\left(u_{1}\right)$ and $P_{\mathrm{tr}}(\omega) \cap W F\left(u_{1}\right)=\phi$.

Theorem 5.2. We assume that $\beta_{2}^{2}<\alpha_{1}^{2} /\left(1-(n(0) \cdot \omega)^{2}\right)$. There exists a function $H_{2}(s)$ whose null points are at most 49 such that if $H_{2}\left(\alpha_{1}^{2} /(1-\right.$ $\left.\left.(n(0) \cdot \omega)^{2}\right)\right) \neq 0, \quad P_{\text {in }}(\omega) \cap W F\left(u_{1}\right)=\left(\tilde{P}_{\text {in }}(\omega) \cup \tilde{S}_{\text {in }}(\omega)\right) \cap W F\left(u_{2}\right)=\phi$ and $S_{i}(\omega) \subset$ $W F\left(u_{1}\right)$, then one of the following two cases occurs: a) $S_{r}(\omega) \subset W F\left(u_{1}\right)$ and 
$\tilde{S}_{r}(\omega) \cup \tilde{P}_{\text {tr }}(\omega) \subset W F\left(u_{2}\right)$. b) $S_{r}(\omega) \subset W F\left(u_{1}\right), \tilde{S}_{r}(\omega) \subset W F\left(u_{2}\right)$ and $\tilde{P}_{\operatorname{tr}}(\omega) \cap W F\left(u_{2}\right)$ $=\phi$. In the above statement is not complete in the following sense: If we suppose that the assumptions mentioned in the above hold and in a small neighbourhood of 0 the border $\Gamma_{0}$ is equal to a hyperplane in $R^{3}$, then we have one of the following two cases; $\left.\mathrm{a}^{\prime}\right)\left(S_{r}(\omega) \cup P_{\mathrm{tr}}(\omega)\right) \subset W F\left(u_{1}\right)$ and $\left.\left(\tilde{S}_{r}(\omega) \cup \tilde{P}_{\mathrm{tr}}(\omega)\right) \subset W F\left(u_{2}\right), \mathrm{b}^{\prime}\right) \quad S_{r}(\omega) \subset W F\left(u_{1}\right), \quad \tilde{S}_{r}(\omega) \subset W F\left(u_{2}\right)$ and $P_{\mathrm{tr}}(\omega) \cap$ $W F\left(u_{1}\right)=\tilde{P}_{\mathrm{tr}}(\omega) \cap W F\left(u_{2}\right)==\phi$.

In order to prove the above theorems we need to change the components of $V_{i}$ in (2.3) corresponding to $S$ waves. Put

$$
\begin{aligned}
h_{1}(s)=\left(\left(\rho_{1}-\rho_{2}\right) s-2\left(\mu-\mu_{2}\right)\right)\left(\rho_{2} s+2\left(\mu_{1}-\mu_{2}\right)\right) & \\
& +2\left(\mu_{1}-\mu_{2}\right)\left(\rho_{1} s-2\left(\mu_{1}-\mu_{2}\right)\right)\left(a_{2} b_{2}\right)(s), \\
h_{2}(s)=\left\{\left(\rho_{1}-\right.\right. & \left.\left.\rho_{2}\right)^{2}-4\left(\mu_{1}-\mu_{2}\right)^{2} /\left(\beta_{1}^{2} \alpha_{2}^{2}\right)\right] s \\
& \quad-4\left(\mu_{1}-\mu_{2}\right)\left\{\left(\rho_{1}-\rho_{2}\right)-\left(\mu_{1}-\mu_{2}\right)\left(\beta_{1}^{-2}+\alpha_{2}^{-2}\right)\right\},
\end{aligned}
$$

where $a_{2}=\left(s / \alpha_{2}^{2}-1\right)^{1 / 2}$ and $b_{2}=\left(s / \beta_{2}^{2}-1\right)^{1 / 2}$, then we have the following

Lemma 5.3. i) If $\beta_{1}^{2}<\alpha_{1}^{2} /\left(1-(n(0) \cdot \omega)^{2}\right)<\beta_{2}^{2}$, then there exists a pseudodifferential operator $a\left(y^{\prime}, D_{y^{\prime}}, D_{t}\right)$ of order 0 such that the principal symbol of $a$ is zero at $y^{\prime}=0$ and that the $(3,2)$ component of $\left(C^{-}\right)^{-1}\left(C_{1}^{+}\right)\left(I_{3}+A\right)$ is 0 modulo $L^{-\infty}\left(\Gamma_{2}\right)$, where $I_{3}$ is the $3 \times 3$ identity matrix and $A\left(y^{\prime}, D_{y^{\prime}}, D_{t}\right)$ is a $3 \times 3$ square matrix whose $(1,2)$ component is a and other components are 0 .

ii) We assume that $\alpha_{1}^{2} /\left(1-(n(0) \cdot \omega)^{2}\right)>\beta_{2}^{2}$. Then there exists a pseudodifferential operator $a\left(y^{\prime}, D_{y^{\prime}}, D_{t}\right)$ such that if $h_{1}\left(\alpha_{1}^{2} /\left(1-(n(0) \cdot \omega)^{2}\right) \neq 0\right.$, (3.2) component of $\left(C^{-}\right)^{-1}\left(C_{1}^{+}\right)\left(I_{3}+A\right)$ is zero modulo $L^{-\infty}\left(\Gamma_{2}\right)$, where $A$ is $a$ similar pseudo-differential operator to that mentioned in i). The similar property on the $(6,2)$ component of $\left(C^{-}\right)^{-1}\left(C_{1}^{+}\right)\left(I_{3}+A\right)$ holds, if $h_{2}\left(\alpha_{1}^{2}(1-\right.$ $\left.(n(0) \cdot \omega))^{2}\right) \neq 0$.

Proof. Define $c_{j}(j=1, \cdots, 6)$ and $c_{j}^{+}(j=1,2,3)$ to be the $j$-th line and column vector of the principal symbol of $\left(C^{-}\right)^{-1}$ and $C_{1}^{+}$, respectively. If we can show that $X={ }^{t} c_{3} \cdot c_{1}^{+}$or $Y={ }^{t} c_{6} \cdot c_{1}^{+}$is not zero at $y^{\prime}=0$, we have the statements of Lemma 5.3 , by using the calculus on symbols of pseudo-differential operators. Let $f_{1}$ be the first column vector of the principal symbol of $C^{-}$. Then $X={ }^{t} c_{3} \cdot\left(c_{1}^{+}-f_{1}\right)$ is equal to $2 a_{1}^{+}\left|\eta^{\prime}\right|^{2} \Lambda_{1}^{-3}\left(c_{31}-\right.$ $\left.2 \mu_{1} c_{36}\right)$ at $y^{\prime}=0$ and $Y={ }^{t} c_{6} \cdot\left(c_{1}^{+}-f_{1}\right)$ is equal to $2 a_{1}^{+}\left|\eta^{\prime}\right|^{2} \Lambda_{1}^{-3}\left(c_{61}-2 \mu_{1} c_{66}\right)$ at $y^{\prime}=0$, where $c_{i j}$ is the $(i, j)$ component of the principal symbol of $\left(C^{-}\right)^{-1}$. 
Using (2.4), we can easily derive that

$$
\begin{gathered}
c_{31}=\left|\eta^{\prime}\right|^{4} \Lambda_{1}^{-8}\left[\left\{\left(\rho_{1}-\rho_{2}\right) \tau^{2}-2\left(\mu_{1}-\mu_{1}\right)\left|\eta^{\prime}\right|^{2}\right\}\left(\rho_{2} \tau^{2}-2 \mu_{2}\left|\eta^{\prime}\right|^{2}\right)\right. \\
\left.\quad-2 \mu_{2} a_{2}^{-} b_{2}^{-}\left\{\left(\rho_{1}-\rho_{2}\right) \tau^{2}-2\left(\mu_{1}-\mu_{2}\right)\left|\eta^{\prime}\right|^{2}\right\}-2 \rho_{2} b_{2}^{-}\left(\mu_{2} a_{2}^{-}-\mu_{1} a_{1}^{-}\right) \tau^{2}\right], \\
c_{36}=-\left|\eta^{\prime}\right|^{4} \Lambda_{1}^{-8}\left[\left\{\left(\rho_{1}-\rho_{2}\right) \tau^{2}-2\left(\mu_{1}-\mu_{2}\right)\left|\eta^{\prime}\right|^{2}\right\}\left(\left|\eta^{\prime}\right|^{2}+a_{2}^{-} b_{2}^{-}\right)-\rho_{2} b_{2}^{-}\left(a_{1}^{-}-a_{2}^{-}\right) \tau^{2}\right], \\
c_{61}=-\left|\eta^{\prime}\right|^{4} \Lambda_{1}^{-8}\left[\left\{\left(\rho_{1}-\rho_{2}\right) \tau^{2}-2\left(\mu_{1}-\mu_{2}\right)\left|\eta^{\prime}\right|^{2}\right\}\left(\rho_{1} \tau^{2}-2 \mu_{1}\left|\eta^{\prime}\right|^{2}\right)\right. \\
\left.\quad+2 \mu_{1} a_{1}^{-} b_{1}^{-}\left\{\left(\rho_{1}-\rho_{2}\right) \tau^{2}-2\left(\mu_{1}-\mu_{2}\right)\left|\eta^{\prime}\right|^{2}\right\}+2 \rho_{1} b_{1}^{-}\left(\mu_{2} a_{2}^{-}-\mu_{1} a_{1}^{-}\right) \tau^{2}\right], \\
c_{66}=-\left|\eta^{\prime}\right|^{4} \Lambda_{1}^{-8}\left[\left\{\left(\rho_{1}-\rho_{2}\right) \tau^{2}-2\left(\mu_{1}-\mu_{2}\right)\left|\eta^{\prime}\right|^{2}\right\}\left(\left|\eta^{\prime}\right|^{2}+a_{1}^{-} b_{1}^{-}\right)-\rho_{1} b_{1}^{-}\left(a_{1}^{-}-a_{2}^{-}\right) \tau^{2}\right] .
\end{gathered}
$$

Thus if $b_{2}^{-}$is pure imaginary, $c_{31}-2 \mu_{1} c_{36}$ does not vanish. When $b_{2}^{-}$is real, $c_{31}-2 \mu_{1} c_{36}$ is not zero at $\rho_{1}$, if $h_{1}\left(\alpha_{1}^{2} /\left(1-(n(0) \cdot \omega)^{2}\right)\right) \neq 0$, and $c_{61}-2 \mu_{1} c_{36}$ is not zero at $\rho_{1}$, if $h_{2}\left(\alpha_{1}^{2} /\left(1-(n(0) \cdot \omega)^{2}\right) \neq 0\right.$. The proof is completed.

Next we shall check the ellipticity of the components of $\left(C^{-}\right)^{-1}\left(C_{1}^{+}\right)$. Put

$$
\begin{aligned}
h_{3}(s)= & \left(\rho_{1} s-2 \mu_{1}\right)\left(\rho_{2} s-2\left(\mu_{1}-\mu_{2}\right)\right) b_{2}(s)+\rho_{1} s\left(\rho_{2} s+2\left(\mu_{1}-\mu_{2}\right)\right) b_{1}(s) \\
& +2 \mu_{1}\left(\rho_{1} s-2\left(\mu_{1}-\mu_{2}\right)\right) a_{2}(s)+4 \mu_{1}\left(\mu_{1}-\mu_{2}\right)\left(a_{1} b_{1}\right)(s)\left(b_{2}-a_{2}\right)(s),
\end{aligned}
$$

where $b_{2}(s)$ is equal to $i \in\left(1-s / \beta_{2}^{2}\right)^{1 / 2}$, if $\beta_{1}^{2}<\alpha_{1}^{2} /\left(1-(n(0) \cdot \omega)^{2}\right)<\beta_{2}^{2}$ and is equal to $\left(s / \beta_{2}^{2}-1\right)^{1 / 2}$, if $\beta_{2}^{2}<\alpha_{1}^{2} /\left(1-(n(0) \cdot \omega)^{2}\right)$. Then we have the following

LemMA 5.4. Let $d_{i j}\left(y^{\prime}, D_{y^{\prime}}, D_{t}\right)$ be the $(i, j)$ component of $\left(C^{-}\right)^{-1} C_{1}^{+}$.

i) When $\beta_{1}^{2}<\alpha_{1}^{2} /\left(1-(n(0) \cdot \omega)^{2}\right)<\beta_{2}^{2}$, $d_{31}$ is elliptic at $\rho_{1}=\left(0, t_{0},-\varepsilon \omega^{\prime}\right.$, $\left.\varepsilon \alpha_{1}\right)$ and $\left(d_{i j}\right)_{i=5, j=1,2}$ is also elliptic at $\rho_{1}$, if $h_{3}\left(\alpha_{1} /\left(1-(n(0) \cdot \omega)^{2}\right) \neq 0\right.$.

ii) When $\left.\beta_{2}^{2}<\alpha_{1}^{2} /(1-n(0) \cdot \omega)^{2}\right), d_{31}$ is elliptic at $\rho_{1}$, if $h_{1}\left(\alpha_{1}^{2} /\left(1-(n(0) \omega)^{2}\right)\right.$ $\neq 0, d_{61}$ is elliptic at $\rho_{1}$, if $h_{1}\left(\alpha_{1}^{2} /\left(1-(n(0) \cdot \omega)^{2}\right) \neq 0\right.$, and $\left(d_{i j}\right)_{i=4,5, j=1,2}$ is elliptic at $\rho_{1}$, if $h_{3}\left(\alpha_{1} /\left(1-(n(0) \cdot \omega)^{2}\right) \neq 0\right.$.

Proof. The ellipticity of $d_{31}$ and $d_{61}$ at $\rho_{1}$ proved in the proof of Lemma 5.3. From (2.4) it follows that the principal symbols of $d_{42}$ and $d_{51}$ are zero at $\rho_{1}$ and the one of $d_{52}$ is not zero at $\rho_{1}$. Thus we may prove that the principal symbol of $d_{41}$ is not zero. By the same way as in the procf of Lemma 5.3, one of equivalent conditions of the ellipticity of $d_{41}$ at $\rho_{1}$ is the principal symbol of $f_{41}-2 \mu_{1} f_{46}$ is not zero at $\rho_{1}$, where $f_{i j}$ is the $(i, j)$ component of the principal symbol of $\left(C^{-}\right)^{-1}$. Making use of (2.4), we can easily derive that $f_{41}-2 \mu_{1} f_{46}$ is equal to $-\left|\eta^{\prime}\right|^{7} \Lambda_{1}^{-7} h_{3}\left(\tau^{2} /\left|\eta^{\prime}\right|^{2}\right)$ at $y^{\prime}=0$. The proof is completed. To prove the theorems we need the following function 


$$
\begin{aligned}
h_{4}(s)= & \left\{\left(\rho_{1}-\rho_{2}\right) s-2\left(\mu_{1}-\mu_{2}\right)\right\}^{2}-\left\{\rho_{2} s+2\left(\mu_{1}-\mu_{2}\right)\right\}^{2}\left(a_{1} b_{1}\right)(s) \\
& +\left\{\rho_{1} s-2\left(\mu_{1}-\mu_{2}\right)\right\}^{2}\left(a_{2} b_{2}\right)(s)-\rho_{1} \rho_{2}\left\{\left(a_{2} b_{1}\right)(s)-\left(a_{1} b_{2}\right)(s)\right\} s^{2} \\
& -4\left(\mu_{1}-\mu_{2}\right)^{2}\left(a_{1} b_{1} a_{2} b_{2}\right)(s),
\end{aligned}
$$

where $a_{i}(s)=\left(s / \alpha_{i}^{2}-1\right)^{1 / 2}(i=1,2), b_{1}(s)=\left(s / \beta_{1}^{2}-1\right)^{1 / 2}$ and $b_{2}(s)$ is $i \varepsilon(1-$ $\left.s / \beta_{2}^{2}\right)^{1 / 2}$ if $\left.\beta_{1}^{2}<\alpha_{1}^{2} /(1-n(0) \cdot \omega)^{2}\right)<\beta_{2}^{2}$ and is $\left(s / \beta_{2}^{2}-1\right)^{1 / 2}$ if $\beta_{2}^{2}<\alpha_{1}^{2} /\left(1-(n(0) \cdot \omega)^{2}\right)$.

Proof of Theorem 5.1. We shall use the same notations appeared in the proof of Theorem 4.1 and $H_{1}(s)=\left(h_{3} h_{4}\right)(s)$. By Lemma 5.3 there exists a pseudo-differential operator $a\left(y^{\prime}, D_{y^{\prime}}, D_{t}\right)$ such that the $(3,2)$ component of $\left(C^{-}\right)^{-1} C_{1}^{+}\left(I_{3}+A\right)$ is essentially zero. We shall put

$$
{ }^{t}\left(\tilde{v}_{11}^{+}, \tilde{v}_{12}^{+}\right)=\left(\begin{array}{ll}
1 & a \\
0 & 1
\end{array}\right)^{-1 t}\left(\tilde{v}_{11}^{+}, \tilde{v}_{12}^{+}\right)
$$

Then they satisfy a hyperbolic equation $\left(D_{y_{3}}-\tilde{A}_{1}^{+}\right)^{t}\left(\tilde{v}_{11}^{+}, \tilde{v}_{12}^{+}\right)=g$ in $y_{3}>0$, where $g$ is smooth at $\rho_{1}$ and the principal symbol $\tilde{A}_{1}^{+}\left(y^{\prime}, D_{y^{\prime}}, D_{t}\right)$ is $\tilde{a}_{1}^{+}\left(y^{\prime}, \eta^{\prime}, \tau\right) I_{2}$. From the assumption the boundary condition in (2.3) is reduced to

$$
\left(\begin{array}{c}
V_{1}^{-} \\
V_{2}^{-}
\end{array}\right)=F\left(y^{\prime}, D_{y^{\prime}}, D_{t}\right)\left(\begin{array}{c}
\tilde{v}_{11}^{+} \\
\tilde{v}_{12}^{+}
\end{array}\right)+G \quad \text { on } y_{3}=0,
$$

where $\rho_{1} \notin W F(G)$ and the first and second column vectors of the $6 \times 2$ matrix $F=\left(c_{i j}\right)$ are equal to these of $-\left(C^{-}\right)^{-1} C_{1}^{+}\left(I_{3}+A\right)$. The assumption $S_{i}(\omega) \subset W F\left(u_{1}\right)$ is equivalent to $\rho_{1} \in W F\left(\left.\tilde{v}_{11}^{+}\right|_{y_{3}=0}\right) \cup W F\left(\left.\tilde{v}_{12}^{+}\right|_{y_{3}=0}\right)$. From Lemma 5.4 it follows that $\rho_{1} \in W F\left(\left.v_{21}^{-}\right|_{y_{3}=0}\right) \cup W F\left(\left.v_{22}^{-}\right|_{y_{3}=0}\right)$, which means $\tilde{S}_{r}(\omega) \subset W F\left(u_{2}\right)$. If we assume that $\rho_{1} \in W F\left(\left.\tilde{v}_{11}^{+}\right|_{y_{3}=0}\right)$, then from the third component of the right hand side of (5.1) we see that $\rho_{1} \in W F\left(\left.v_{13}^{-}\right|_{y_{3}=0}\right)$, that is $P_{\mathrm{tr}}(\omega) W F\left(u_{1}\right)$. On the other hand if $\rho_{1} \notin W F\left(\left.\tilde{v}_{11}^{+}\right|_{y_{3}=0}\right)$, then by the same reason it follows that $\rho_{1} \notin W F\left(\left.v_{13}\right|_{y_{3}=0}\right)$, that is $P_{\mathrm{tr}}(\omega) \cap W F\left(u_{1}\right)=\phi$. Finally we shall show that $\rho_{1} \in W F\left(\left.v_{11}^{-}\right|_{y_{3}=0}\right) \cup W F\left(\left.v_{12}^{-}\right|_{y_{3}=0}\right)$, if $h_{4}\left(\alpha_{1}^{2} /\left(1-(n(0) \cdot \omega)^{2}\right) \neq 0\right.$. We assume $\rho_{1} \notin W F\left(\left.v_{11}^{-}\right|_{y_{3}=0}\right) \cup W F\left(\left.v_{12}^{-}\right|_{y_{3}=0}\right)$, then from the assumptions it follows that does not belong to the wave front set of $\left.F_{1}\left(y^{\prime}, D_{y^{\prime}}, D_{t}\right)^{t}\left(\tilde{v}_{11}^{+}, \tilde{v}_{12}^{+}, v_{13}^{-},{ }^{t} V_{2}\right)\right|_{y_{3}=0}$, where the first and second column vectors of $F_{1}$ are equal to these of $C_{1}^{+}\left(I_{3}+A\right)$, the third column vector of $F_{1}$ is equal to one of $C_{1}^{-}$, and the fourth, fifth and sixth column vectors of $F_{1}$ are equal to these of $-C_{2}$. If $F_{1}$ is elliptic at $\rho_{1}$, we have $\rho_{1}$ does not belong to $W F\left(\left.\tilde{v}_{11}^{+}\right|_{y_{3}=0}\right) \cup W F\left(\left.\tilde{v}_{12}^{+}\right|_{y_{3}=0}\right)$. This is a contradiction. From $(2.4)$ if $h_{4}\left(\alpha_{1}^{2} /\left(1-(n(0) \cdot \omega)^{2}\right)\right.$ is not zero, $F_{1}$ is elliptic at $\rho_{1}$. The proof is completed. Next we shall consider the case $\beta_{2}^{2}<\alpha_{1}^{2} /\left(1-(n(0) \cdot \omega)^{2}\right)$. 
Proof of Theorem 5.2. We put $H_{2}(s)=\left(h_{2} h_{3} h_{4}\right)(s)$. Using Lemma 5.3, we can prove the first part by the same way as in the proof of Theorem 5.1. If we assume that $\Gamma_{0}$ is flat near 0 , then the reduced boundary value problem (2.3) does not depend on $y^{\prime}$. Thus the symbol of $C_{i}^{ \pm}\left(D_{y^{\prime}}, D_{t}\right)$ is given by $(2.4)$. It follows that the $(3,2)$ and $(6,2)$ components of $\left(C^{-}\right)^{-1} C_{1}^{+}$is both zero. Using this fact, we can prove the later part of Theorem 5.1.

In the statement of the first part of Theorem 5.2 we only consider refracted singularities, however on reflected singularities we have the following

Remark 5.5. In assumptions of Theorem 5.2 we assume $\left(h_{1} h_{3} h_{4}\right)\left(\alpha_{1}^{2} /(1-\right.$ $\left.(n(0) \cdot \omega))^{2}\right) \neq 0$ instead of $\left(h_{2} h_{3} h_{4}\right)\left(\alpha_{1}^{2} /(1-(n(0) \cdot \omega))^{2}\right) \neq 0$. Then making use of the statement ii) of Lemma 5.3 we have the following two cases: $a^{\prime \prime}$ ) $S_{r}(\omega) \cup P_{\mathrm{tr}}(\omega) \subset W F\left(u_{1}\right)$ and $\left.\tilde{S}_{r}(\omega) \subset W F\left(u_{2}\right) . \quad \mathrm{b}^{\prime \prime}\right) S_{r}(\omega) \subset W F\left(u_{1}\right), \quad \tilde{S}_{r}(\omega) \subset$ $W F\left(u_{2}\right)$ and $P_{\operatorname{tr}}(\omega) \cap W F\left(u_{1}\right)=\phi$.

\section{REFERENCES}

[1] G. Duvaut and J. L. Lions, Inequalities in mechanics and physics, Grundlehren d. Math. Wiss., 219, Springer-Verlag, 1976.

[2] L. Hörmander, Linear partial differential operators, Grundlehren d. Math. Wiss., 116, Springer-Verlag, 1963.

[ 3 ] — - Fourier integral operator I, Acta Math., 127 (1971), 79-183.

[4] — Fourier integral operators II, Acta Math., 128 (1972), 183-269.

[5] L. Nirenberg, Lectures on linear partial differential equations, Regional Conferences Series in Math., 17, Providence 4, R.I., 1973.

[6 ] R. Melrose and J. Sjostrand, Singularities of boundary value problems I, Comm. Pure Appl. Math., 31 (1978), 593-617.

[ 7 ] R. Stoneley, Elastic waves at the surface of separation of two solids, Proc. Roy. Soc. London, 106 (1924), 416-428.

[8] M. E. Taylor, Reflection of singularities of solutions to systems of differential equations, Comm. Pure Appl. Math., 28 (1975), 457-478.

[ 9 ] K. Yamamoto, Singularities of solutions to the boundary value problems for elastic and Maxwell's equations, Japanese J. Math., 14 (1988), 119-163.

[10] —- Reflective elastic waves at the boundary as a propagation of singularities phenomenon, (to appear).

[11] C. H. Wilcox, Scattering Theory for the d'Alembert Equation in Exterior Domains, Lecture Notes in Math., 442, Springer-Verlag, 1975.

Department of Mathematics

Nagoya Institute of Technology

Nagoya 466, Japan 